\title{
Resolved molecular line observations reveal an inherited molecular layer in the young disk around TMC1 $A^{\star}$
}

\author{
D. Harsono ${ }^{1,2}$, M. H. D. van der Wiel ${ }^{3}$, P. Bjerkeli ${ }^{4}$, J. P. Ramsey ${ }^{5}$, H. Calcutt ${ }^{4}$, L. E. Kristensen ${ }^{6}$, and J. K. Jørgensen ${ }^{6}$ \\ ${ }^{1}$ Leiden Observatory, Leiden University, Niels Bohrweg 2, 2300 RA Leiden, The Netherlands \\ e-mail: harsono@strw. leidenuniv.nl \\ ${ }^{2}$ Institute of Astronomy and Astrophysics, Academia Sinica, No. 1, Sec. 4, Roosevelt Road, Taipei 10617, Taiwan, PR China \\ 3 ASTRON, the Netherlands Institute for Radio Astronomy, Oude Hoogeveensedijk 4, 7991 PD Dwingeloo, The Netherlands \\ ${ }^{4}$ Chalmers University of Technology, Onsala Space Observatory, 43992 Onsala, Sweden \\ ${ }^{5}$ Department of Astronomy, University of Virginia, Charlottesville, VA 22904, USA \\ ${ }^{6}$ Niels Bohr Institute and Centre for Star and Planet Formation, University of Copenhagen, Øster Voldgade 5-7, \\ 1350 Copenhagen K, Denmark
}

Received 17 June 2020 / Accepted 23 October 2020

\begin{abstract}
Context. Physical processes that govern the star and planet formation sequence influence the chemical composition and evolution of protoplanetary disks. Recent studies allude to an early start to planet formation already during the formation of a disk. To understand the chemical composition of protoplanets, we need to constrain the composition and structure of the disks from whence they are formed. Aims. We aim to determine the molecular abundance structure of the young disk around the TMC1A protostar on au scales in order to understand its chemical structure and any possible implications for disk formation.

Methods. We present spatially resolved Atacama Large Millimeter/submillimeter Array observations of $\mathrm{CO}, \mathrm{HCO}^{+}, \mathrm{HCN}, \mathrm{DCN}$, and SO line emission, as well as dust continuum emission, in the vicinity of TMC1A. Molecular column densities are estimated both under the assumption of optically thin emission from molecules in local thermodynamical equilibrium (LTE) as well as through more detailed non-LTE radiative transfer calculations.

Results. Resolved dust continuum emission from the disk is detected between 220 and $260 \mathrm{GHz}$. Rotational transitions from $\mathrm{HCO}^{+}$, $\mathrm{HCN}$, and SO are also detected from the inner 100 au region. We further report on upper limits to vibrational $\mathrm{HCN} v_{2}=1$, DCN, and $\mathrm{N}_{2} \mathrm{D}^{+}$lines. The $\mathrm{HCO}^{+}$emission appears to trace both the Keplerian disk and the surrounding infalling rotating envelope. $\mathrm{HCN}$ emission peaks toward the outflow cavity region connected with the CO disk wind and toward the red-shifted part of the Keplerian disk. From the derived $\mathrm{HCO}^{+}$abundance, we estimate the ionization fraction of the disk surface, and find values that imply that the accretion process is not driven by the magneto-rotational instability. The molecular abundances averaged over the TMC1A disk are similar to its protostellar envelope and other, older Class II disks. We meanwhile find a discrepancy between the young disk's molecular abundances relative to Solar System objects.

Conclusions. Abundance comparisons between the disk and its surrounding envelope for several molecular species reveal that the bulk of planet-forming material enters the disk unaltered. Differences in $\mathrm{HCN}$ and $\mathrm{H}_{2} \mathrm{O}$ molecular abundances between the disk around TMC1A, Class II disks, and Solar System objects trace the chemical evolution during disk and planet formation.
\end{abstract}

Key words. stars: formation - stars: protostars - ISM: abundances - astrochemistry - ISM: individual objects: TMC1A protoplanetary disks

\section{Introduction}

The properties of newborn planets will likely reflect the environment in which they formed. Thus, knowing the physical and chemical structure of protoplanetary disks is absolutely necessary in order to understand the process of planet formation. Evidence for on-going planet formation has been captured by recent high-spatial resolution millimeter and high-contrast IR observations that reveal gaps, rings, and spiral dust substructures in protoplanetary disks (e.g., ALMA Partnership et al. 2015; Pérez et al. 2016; Long et al. 2018; Huang et al. 2018). The immediate emerging paradigm is that planet formation starts early during the embedded stage of star formation (Class 0 and I, e.g., Harsono et al. 2018). This is supported by cosmochemical

\footnotetext{
* The spectral cubes (FITS) are only available at the CDS via anonymous ftp to cdsarc.u-strasbg.fr $(130.79 .128 .5)$ or via http://cdsarc.u-strasbg.fr/viz-bin/cat/J/A+A/646/A72
}

measurements of calcium aluminium inclusions and chondrules that are found in chondritic meteorites formed in the early Solar System (Connelly et al. 2012; Bollard et al. 2017; Jacquet et al. 2019; Haugbølle et al. 2019). A spatially resolved molecular studies of a young disk can reveal the environment of the early stages of planet formation.

Understanding the physical and chemical structure of planetforming disks relies on both high-spatial resolution and spectrally resolved observations. From dust continuum observations, the physical structure of Class II disks have been constructed and compared to theoretical studies of disk evolution (Williams \& Cieza 2011; Manara et al. 2017, 2019; Tazzari et al. 2017). Once a physical structure is determined, the chemical structure can be studied and compared with evolutionary models coupled to chemistry (e.g., Dutrey et al. 1997; van Zadelhoff et al. 2001; Aikawa et al. 2003; Ceccarelli et al. 2005; Piétu et al. 2007; Bergin et al. 2010; Bruderer et al. 2012; Chapillon et al. 2012; 
van der Marel et al. 2014; Loomis et al. 2015; Guzmán et al. 2015; Fedele et al. 2016; Salinas et al. 2016; Kama et al. 2016; McClure et al. 2016; Öberg et al. 2017; Carney et al. 2018). Meanwhile, ongoing planet formation can also affect the strength of molecular line emission, as suggested by a decreasing $\mathrm{HCN} / \mathrm{H}_{2} \mathrm{O}$ midIR line flux ratio with decreasing disk mass observed toward Class II disks (Najita et al. 2013). However, similar studies towards embedded, young stellar objects are still difficult.

Detailed studies of the physical and chemical structure of young, embedded disks are hindered by the surrounding, obscuring protostellar envelope. In addition, since the chemical evolutionary timescale in the disk is typically longer than the envelope infall timescale, the final chemical structure of the disk also depends on the physical and chemical evolution of the protostellar envelope (Bergin \& Langer 1997; Aikawa et al. 1999; Visser et al. 2009; Hincelin et al. 2013; Yoneda et al. 2016). In this regard, chemical studies with single-dish telescopes have been instrumental in determining the bulk chemical structure of large-scale envelopes around low-mass stars (>1000 au, e.g., Blake et al. 1995; van Dishoeck \& Blake 1998; Jørgensen et al. 2004; Graninger et al. 2016) down to the scales of a few hundred au with millimeter interferometers such as the Submillimeter Array and IRAM NOrthern Extended Millimeter Array (e.g., Jørgensen et al. 2005, 2007; Bisschop et al. 2008; Maury et al. 2014; Taquet et al. 2015). With the Atacama Large Millimeter/submillimeter Array, it is now possible to spatially and spectrally resolve the molecular emission to isolate the Keplerian disk from the surrounding envelope (e.g., Sakai et al. 2014a; Artur de la Villarmois et al. 2018, 2019b). This aspect makes it possible to explore the chemical structure of young disks and examine the early stages of planet formation.

Many inferred chemical compositions demonstrate a link between Solar System comets and young stellar objects, while others indicate differences since formation (e.g., Schöier et al. 2002; Visser et al. 2009; Pontoppidan et al. 2014; Persson et al. 2014; Garrod 2019). For example, the most recent comparison between the young stellar object IRAS 16293-2422 and comet 67P/Churyumov-Gerasimenko shows a correlation in the CHO-, $\mathrm{N}$ - and S-bearing molecules (Drozdovskaya et al. 2019). Meanwhile molecular species such as $\mathrm{CH}_{3} \mathrm{OH}, \mathrm{CH}_{3} \mathrm{CN}$, and $\mathrm{CH}_{3} \mathrm{SH}$ are elevated compared to comets, which indicates some degree of processing. Further evidence for a direct link between the birth environment of a planet and the initial structure of the proto-Solar disk can be found in the elemental enhancement of Jupiter's atmosphere with respect to solar (Owen et al. 1999; Asplund et al. 2009; Öberg \& Wordsworth 2019; Bosman et al. 2019). It is therefore crucial to understand the chemistry and variation of molecular abundances during the early stages of disk evolution to be able to draw conclusions about the details of planetary composition.

In the classical theory of a disk (Lodato 2008; Armitage 2011), a protoplanetary disk is characterized by its Keplerian motion. Hence, molecular emission emission from a rotationally supported object will more likely trace the chemical structure of an early disk. A few young stellar objects show a clear sign of an embedded Keplerian disk (e.g. Jørgensen et al. 2009; Harsono et al. 2014; Yen et al. 2017; Artur de la Villarmois et al. 2019a). Only a handful of these targets are less embedded and at a favorable orientation such that there is a direct line-of-sight to the disk through the tenuous envelope. One of those targets is TMC1A (IRAS 04365+2535) with $i \sim 60^{\circ}$ and a $\sim 0.1 M_{\odot}$ envelope, which makes it a "Stage I" embedded young stellar object (Robitaille et al. 2006; Kristensen et al. 2012). A "Stage I" system refers to a protostellar object with an envelope mass that is similar to its protostellar mass. High-spatial resolution observations of ${ }^{12} \mathrm{CO},{ }^{13} \mathrm{CO}$, and $\mathrm{C}^{18} \mathrm{O}$ demonstrate a clear distinction between outflowing gas (Bjerkeli et al. 2016), the Keplerian disk, and the large-scale envelope (Harsono et al. 2018). The relatively simple geometry of TMC1A with its well-defined disk, makes it an ideal laboratory for determining the distribution and abundances of common, simple molecules on scales of $\sim 15 \mathrm{au}$. This paper presents such an analysis and compares the inferred chemistry to that of the Solar System as well as more evolved young stellar objects. Thereby it also helps shedding light on which molecules can serve as fingerprints of disk formation and evolution.

This paper presents the detection of simple molecules in the young disk around TMC1A. By determining their molecular column densities, we constrain the molecular abundance structure of its Keplerian disk with respect to $\mathrm{C}^{18} \mathrm{O}$. The paper outline is as follows. Section 2 presents the observational details. Dust continuum emission and the detected molecular lines are presented in Sect. 3. HCN and DCN lines are analyzed further using the $\mathrm{C}^{18} \mathrm{O}$ spectral cube as a proxy mask. In Sect. 4, optically thin, thermalized molecular emission and non-local thermodynamical equilibrium (LTE) radiative transfer calculations are been used to determine the molecular column densities and their excitation conditions. Using these column densities, we estimate the molecular abundances and temperature structure of the young disk around TMC1A. By utilizing a reduced chemical network, the ionization fraction of the disk is approximated in order to understand the physical processes that drive its accretion. The derived abundances are compared with the protostellar envelope, Class II protoplanetary disks, and Solar System objects in Sect. 5. Finally, the summary and conclusions can be found in Sect. 6.

\section{Observations}

TMC1A was observed during long baselines campaigns (LBC, $16 \mathrm{~km}$ ) of the Atacama Large Millimeter/submillimeter Array (ALMA) on three occasions. We have concatenated three LBC projects: 2015.1.01549.S (PI: van der Wiel), 2016.1.00711.S (PI: van der Wiel), and 2017.1.00212.S (PI: Bjerkeli). The first data set (2015.1.01549.S, tuned to frequencies of CO and its isotopologs) was published in Bjerkeli et al. (2016) and Harsono et al. (2018). We also use water $\left(\mathrm{H}_{2}^{18} \mathrm{O} 3_{1,3}-2_{2,0}\right.$ at $\left.203 \mathrm{GHz}\right)$ observations taken with the NOrthern Extended Millimeter Array (NOEMA), and which are presented in Harsono et al. (2020). Here, we present observational details of the second (2016.1.00711.S, Sect. 2.1) and third (2017.1.00212.S, Sect. 2.2) ALMA data sets, while we refer to Harsono et al. $(2018,2020)$ for details on the imaging and calibration of the first ALMA data set and the NOEMA data, respectively. Table 1 lists the observational details of the ALMA programs used in this paper.

\subsection{ALMA observations: 2016.1.00711.S}

ALMA observed TMC1A on September 22 and 23 in 2017. The observations were taken with $\sim 40$ antennas under very good weather conditions with a precipitable water vapor (PWV) of $0.35 \mathrm{~mm}$. The baseline coverage was between $40 \mathrm{~m}$ and $12 \mathrm{~km}$, which translates to $30 \mathrm{k} \lambda$ up to $10^{4} \mathrm{k} \lambda$. The bandpass, phase, and flux calibrators are indicated in Table 1. Four spectral windows were utilized in this program. Three narrow high resolution windows were centered on $\mathrm{HCN} J=3-2$ $(265.8861800 \mathrm{GHz}), \mathrm{HCO}^{+} J=3-2(267.8527094 \mathrm{GHz})$, and 
Table 1. ALMA observational details on Band 6 long baseline observations of TMC1A.

\begin{tabular}{|c|c|c|c|c|c|c|c|}
\hline Date & Bandpass & Phase & Flux & Bl. coverage $(\mathrm{km})$ & $N_{\text {ant. }}$ & PWV (mm) & WVR corr. ${ }^{(a)}$ \\
\hline \multicolumn{8}{|c|}{ 2015.1.01549.S } \\
\hline Oct. 16,2015 & $\mathrm{~J} 0510+1800$ & $\mathrm{~J} 0440+2728$ & $\mathrm{~J} 0433+2905$ & $0.04-16.2$ & 42 & 1.8 & Yes \\
\hline Oct. 23,2015 & $\mathrm{~J} 0510+1800$ & J0440+2728 & J0433+2905 & $0.04-16.2$ & 40 & 0.6 & Yes \\
\hline Oct. 30, 2015 & $\mathrm{~J} 0510+1800$ & $\mathrm{~J} 0440+2728$ & J0433+2905 & $0.04-16.2$ & 40 & 0.3 & Yes \\
\hline \multicolumn{8}{|c|}{ 2016.1.00711.S } \\
\hline Sep. 22, 2017 & $\mathrm{~J} 0510+1800$ & J0438+3004 & $\mathrm{J} 0510+1800$ & $0.04-12.1$ & 42 & 0.7 & No \\
\hline Sep. 23, 2017 & $\mathrm{~J} 0510+1800$ & $\mathrm{~J} 0438+3004$ & $\mathrm{~J} 0510+1800$ & $0.04-12.1$ & 40 & 0.4 & No \\
\hline \multicolumn{8}{|c|}{ 2017.1.00212.S } \\
\hline Oct. 19, 2017 & $\mathrm{~J} 0510+1800$ & J0438+3004 & $\mathrm{J} 0510+1800$ & $0.04-16.2$ & 51 & 1.1 & No \\
\hline
\end{tabular}

Notes. Project IDs are indicated. ${ }^{(a)}$ Application of the non-standard WVR correction as presented in Maud et al. (2017).

SO $N_{J}=5_{6}-4_{5}(251.82577000 \mathrm{GHz})$, respectively. A wide spectral window $(1.8 \mathrm{GHz})$ was centered at $253.2 \mathrm{GHz}$ to measure the dust continuum. Rest line frequencies are obtained from the JPL (Pickett et al. 1998) and CDMS catalogs (Müller et al. 2005; Endres et al. 2016). The data were calibrated using the Cycle 4 pipeline of CASA 4.7.2 (McMullin et al. 2007).

\subsection{ALMA observations: 2017.1.00212.S}

This ALMA project was executed on October 19 2017. The observations were taken with $\sim 51$ antennas. The baseline coverage was between $40 \mathrm{~m}$ and $16.2 \mathrm{~km}$, which translates to $30-10^{4} \mathrm{k} \lambda$. The bandpass, phase, and flux calibrators are also indicated in Table 1. Four broadband continuum spectral windows $(1.8 \mathrm{GHz})$ were used in this program. Molecular lines are not clearly detected because of the low spectral resolution. The data was calibrated using the Cycle 5 pipeline of CASA 5.1.1.

\subsection{Self-calibration and combined ALMA data}

We combined these ALMA projects into a single measurement set using the CASA task CONCAT to improve the signal-to-noise ratio $(\mathrm{S} / \mathrm{N})$ of the dust continuum model. The concatenated data allows for simultaneous phase and amplitude self-calibrations to produce better flux calibration across the three data sets. This self-calibration is based on the line-free channels in each spectral window, and was performed with CASA 5.4.1. We fixed the phase center of each measurement to the value obtained in Harsono et al. (2018) utilizing the data with the most stable weather conditions. The phase center of J2000 04h39m35.203s $+25 \mathrm{~d} 41 \mathrm{~m} 44.21 \mathrm{~s}$ was determined by fitting an elliptical Gaussian to the continuum visibilities with CASA task UVFIT. The phase solutions obtained from the broadband windows are applied across the narrow spectral windows. The imaging of the continuum is performed with TCLEAN using Briggs weighting (Briggs 1995) to minimize the side lobes. Spectral windows containing the targeted molecular lines are continuum subtracted in the $(u, v)$ plane with the task UVCONTSUB.

The targeted molecular lines are listed in Table 2 along with the noise level per spectral resolution element. The spectral lines are imaged at $0.3 \mathrm{~km} \mathrm{~s}^{-1}$ velocity resolution including a spatial tapering at $0{ }^{\prime} 08$, resulting in the synthesized beam sizes indicated in Table 2. Appendix A lists a few molecular lines that were present in our spectral set up, but not detected in our data sets.

\section{Observational results}

\subsection{Spatially resolved dust continuum}

The continuum images of TMC1A between 203 and $260 \mathrm{GHz}$ from the NOEMA and ALMA observations are shown in Fig. 1. The NOEMA observation does not spatially resolve the disk, which is evidenced by the lack of elongation present in the ALMA images. However, the deconvolved size $\left(0{ }^{\prime} 56 \times 00^{\prime} 44\right)$, as determined by fitting an elliptical Gaussian to the observed visibilities, indicates that the dust continuum is mostly tracing the Keplerian disk ( 100 au, Harsono et al. 2014). The peak intensities of the different continuum images are $93 \mathrm{mJy}^{\text {beam }}{ }^{-1}$ at $203 \mathrm{GHz}, 5.1 \mathrm{mJy}$ beam $^{-1}$ at $242 \mathrm{GHz}, 5 \mathrm{mJy} \mathrm{beam}^{-1}$ at $240 \mathrm{GHz}$, and $9.8 \mathrm{mJy}^{\text {beam }}{ }^{-1}$ at $260 \mathrm{GHz}$.

Continuum visibilities at various frequencies are shown in Fig. 2 including the Sub-millimeter Array (SMA) data from Jørgensen et al. (2009) and NOEMA data from Harsono et al. (2014). The binned SMA data are scaled to $1.3 \mathrm{~mm}$ using a frequency dependence of $S_{v} \propto v^{2.5}$. The phase as a function of projected baselines is close to zero for the ALMA observations, indicating the high data quality. The large standard deviation in the NOEMA data reflects the lower number of $(u, v)$ points relative to the ALMA data. From the comparison between the integrated flux density of the NOEMA and ALMA data at $220 \mathrm{GHz}$, the uncertainty in the absolute flux density is within $10 \%$. It is interesting to note that the amplitude of the $260 \mathrm{GHz}$ observations is lower than the values at $220 \mathrm{GHz}$ at short baselines $(<1000 \mathrm{k} \lambda)$ while the opposite is true at long baselines $(>1000 \mathrm{k} \lambda)$. The peak intensity of the dust continuum image at $260 \mathrm{GHz}$ is indeed higher than the peak at the lower frequencies. However, as indicated in Table 2, the integrated continuum flux density at $260 \mathrm{GHz}$ is lower than at $203 \mathrm{GHz}$ in the image plane. The lower flux density at $260 \mathrm{GHz}$ may indicate resolved-out continuum emission at the higher frequency.

To characterize the dust disk, we utilize GALARIO (Tazzari et al. 2018); GALARIO calculates the synthetic $(u, v)$ visibilities given an intensity profile and makes it possible to fit Gaussian intensity profiles to the observed visibilities while simultaneously constraining the flux density and the deconvolved size. The free parameters are the peak intensity $I$ in $\mathrm{Jy}^{-1}$, size of the emitting region, inclination $i$, position angle PA, and position offset. The Markov chain Monte Carlo (MCMC) Python package EMCEE (Foreman-Mackey et al. 2013) is used to efficiently explore a wide range of parameters. At each observed frequency, a first run is performed with 60 walkers $(10 \times$ free 
Table 2. Millimeter interferometric observations presented in this paper.

\begin{tabular}{|c|c|c|c|c|c|c|c|}
\hline Name & $\begin{array}{c}\text { Frequency } \\
(\mathrm{GHz})\end{array}$ & $\begin{array}{l}E_{\mathrm{up}} \\
(\mathrm{K})\end{array}$ & $\begin{array}{c}\log _{10} A_{\mathrm{ul}} \\
\quad\left(\mathrm{s}^{-1}\right)\end{array}$ & $\begin{array}{l}\operatorname{Beam}^{(a)} \\
\left({ }^{\prime \prime} \times{ }^{\prime \prime},{ }^{\circ}\right)\end{array}$ & $\begin{array}{c}\text { Noise } \\
\left(\mathrm{mJy} \mathrm{bm}^{-1}\right)\end{array}$ & $\begin{array}{l}\text { Int. time } \\
(\min )\end{array}$ & $\begin{array}{l}\text { Integrated flux density } \\
\left(\mathrm{mJy} / \mathrm{mJy} \mathrm{km} \mathrm{s}^{-1}\right)\end{array}$ \\
\hline NOEMA continuum & 203 & $\ldots$ & $\ldots$ & $0.78 \times 0.72,62$ & 1.1 & 180 & $210 \pm 20$ \\
\hline NOEMA continuum & 220 & $\ldots$ & $\ldots$ & $0.79 \times 0.61,54$ & 1.2 & 180 & $170 \pm 17$ \\
\hline ALMA continuum & 220 & $\ldots$ & $\ldots$ & $0.031 \times 0.020,-3$ & 0.08 & 170 & $220 \pm 22$ \\
\hline ALMA continuum & 230 & $\cdots$ & $\cdots$ & $0.032 \times 0.021,1$ & 0.05 & 111 & $240 \pm 24$ \\
\hline ALMA continuum & 240 & $\ldots$ & $\ldots$ & $0.028 \times 0.018,0$ & 0.08 & 90 & $280 \pm 28$ \\
\hline ALMA continuum & 260 & $\ldots$ & $\ldots$ & $0.049 \times 0.032,11$ & 0.05 & 63 & $190 \pm 19$ \\
\hline $\mathrm{H}_{2}^{18} \mathrm{O} 3_{1,3}-2_{2,0}$ & 203.4075 & 203.7 & -5.32 & $0.78 \times 0.72,59$ & 8 & 180 & $<11$ \\
\hline DCN 3-2 & 217.2385 & 20.85 & -3.34 & $0.13 \times 0.10,32$ & 1.5 & 91 & $<8$ \\
\hline $\mathrm{C}^{18} \mathrm{O} 2-1$ & 219.5604 & 15.80 & -6.22 & $0.13 \times 0.10,31$ & 1.8 & 91 & $330 \pm 33$ \\
\hline${ }^{13} \mathrm{CO} 2-1$ & 220.3987 & 15.87 & -6.22 & $0.13 \times 0.10,32$ & 2.4 & 85 & $290 \pm 30$ \\
\hline${ }^{12} \mathrm{CO} 2-1$ & 230.5380 & 16.60 & -6.16 & $0.12 \times 0.10,0.5$ & 2.5 & 83 & $1740 \pm 174$ \\
\hline $\mathrm{N}_{2} \mathrm{D}_{+} J=3-2$ & 231.3216 & 22.20 & -2.67 & $0.12 \times 0.10,32$ & 0.8 & 91 & $100 \pm 22$ \\
\hline SO $N=65-5_{4}$ & 251.8258 & 50.66 & -3.71 & $0.13 \times 0.11,0.1$ & 1.0 & 71 & $460 \pm 46$ \\
\hline $\mathrm{HCN} v_{2}=1 J=3-2 e$ & 265.8527 & 1050 & -2.64 & $0.092 \times 0.07,0.4$ & 0.9 & 71 & $<20$ \\
\hline HCN 3-2 & 265.8862 & 25.52 & -3.55 & $0.12 \times 0.10,0.1$ & 1.3 & 71 & $160 \pm 20$ \\
\hline $\mathrm{HCN} v_{2}=1 J=3-2 f$ & 267.1993 & 1050 & -2.63 & $0.092 \times 0.07,20$ & 0.9 & 71 & $<20$ \\
\hline $\mathrm{HCO}^{+} J=3-2$ & 267.5576 & 25.68 & -2.84 & $0.12 \times 0.11,3.5$ & 1.2 & 71 & $640 \pm 64$ \\
\hline
\end{tabular}

Notes. Synthesized beams and noise levels of the images are listed below. Upper limits are calculated over the size of the dust continuum emission. We report the noise to be $10 \%$ of the integrated flux density unless the measured noise is larger than $10 \%$. The noise level for the molecular line observations is per velocity channel $\left(0.3 \mathrm{~km} \mathrm{~s}^{-1}\right) .{ }^{(a)}$ Elliptical synthesized beam parametrized by the full-width half-maximum (FWHM) of the major axis $\times$ the FWHM of the minor axis, and a position angle.



Fig. 1. Dust continuum images at various frequencies. The color scale is shown on the top of each panel starting at $5 \sigma$. The white contours indicate the $5,20,35, \ldots \sigma$ contours up to the peak intensity. A scale of 100 au is shown in the first two panels for reference. The synthesized beam is indicated by a white ellipse in the bottom left corner of each panel. $a$ : NOEMA observations at $203 \mathrm{GHz}$. The color scale spans linearly from 0 to the maximum intensity of $93 \mathrm{mJy}$ beam $^{-1}$. The square box indicates the $1^{\prime \prime} \times 1^{\prime \prime}$ region of the ALMA data that is shown in panels $b, c$ and $d$. $b$ : dust continuum image of the $240 \mathrm{GHz}$ ALMA data. The color scale spans up to the maximum intensity of $5 \mathrm{mJy}^{-1}$ beam $^{-1}$. $c$ : dust continuum image of the $260 \mathrm{GHz}$ ALMA data. The color scale spans from 0 to the maximum intensity of $9.8 \mathrm{mJy} \mathrm{beam}^{-1}$. $d$ : dust continuum emission of the combined ALMA data imaged at $242 \mathrm{GHz}$. Similarly, the color scale spans from 0 to the maximum intensity of $5.1 \mathrm{mJy}^{-1}$ beam ${ }^{-1}$.

parameters) and 1000 steps. Each walker explores the parameter space by gradually stepping into a region with the lowest $\chi^{2}$. The best-fit values are obtained by calculating the mean of the last 100 steps of all walkers. Then, a second run is performed with 120 walkers initialized by uniformly distributing them around the best-fit values obtained from the first run. This second run is performed with 10000 steps to obtain the final set of best-fit parameters. The best-fit values are determined statistically taking the mean of the last 1000 steps of the walkers with acceptance ratios greater than $20 \%$. These best-fit values and their $1 \sigma$ errors are listed in Table 3.

The MCMC modelling of this high-fidelity data allow us to accurately determine that the TMC1A protostellar system is inclined at $50^{\circ} \pm 3$ with a position angle of $75^{\circ} \pm 4$. The size of the dust disk is of the order of 0.1 ( $F W H M=0.23$ or $30 \mathrm{au}$ at $140 \mathrm{pc})$. The deconvolved dust disk size determined from the high angular resolution ALMA data is smaller than the 100 au radius gaseous Keplerian disk (Harsono et al. 2014). Most of the dust continuum flux density is emitted from the region probed by our ALMA data since the difference between the flux density obtained by ALMA and NOEMA data is small $(<20 \%)$ at $220 \mathrm{GHz}$.

\subsection{Molecular gas observations}

In Fig. 3, we present spectra of the targeted molecular lines integrated over a $1^{\prime \prime}$ box centered on TMC1A. Out of the many targeted molecular lines $\left({ }^{12} \mathrm{CO} 2-1,{ }^{13} \mathrm{CO} 2-1, \mathrm{C}^{18} \mathrm{O} 2-1\right.$, DCN 




Fig. 2. Dust continuum amplitudes and phases as a function of projected baselines $R_{\mathrm{uv}}$ in $k \lambda$. Different colors and symbols show the various data sets used. The black, square box near the axis shows the $850 \mu \mathrm{m}$ single-dish flux density scaled to $1.1 \mathrm{~mm}$ (see Harsono et al. 2014). The long baseline ALMA data are split into four different frequencies: 220, 230, 240 and $260 \mathrm{GHz}$ (see Table 2). The standard deviation of each radial bin is also shown, while the corresponding physical scale in au is indicated on the top axis.

Table 3. Best-fit parameters for the Gaussian intensity profile model.

\begin{tabular}{cccccc}
\hline \hline Name & $\begin{array}{c}\text { Size } \\
\left({ }^{\prime \prime}\right)\end{array}$ & $\begin{array}{c}i \\
\left({ }^{\circ}\right)\end{array}$ & $\begin{array}{c}\text { PA } \\
\left({ }^{\circ}\right)\end{array}$ & $\begin{array}{c}\text { Offset } \\
\left({ }^{\prime \prime},,^{\prime \prime}\right.\end{array}$ & $\begin{array}{c}\text { Flux density } \\
(\mathrm{mJy})\end{array}$ \\
\hline NOEMA 203 & $\ldots$ & $\ldots$ & $\ldots$ & $\ldots$ & $\ldots$ \\
NOEMA 220 & $0.2 \pm 0.1$ & $45_{-30}^{+26}$ & $80_{-50}^{+60}$ & $0,-0.13$ & $250_{-2100}^{+2100}$ \\
ALMA 220 & $0.1 \pm 0.01$ & $52 \pm 3$ & $76 \pm 5$ & $0.01,-0.02$ & $300_{-16}^{+18}$ \\
ALMA 230 & $0.1 \pm 0.01$ & $51 \pm 3$ & $75 \pm 3$ & $0.01,0.001$ & $310_{-11}^{+12}$ \\
ALMA 240 & $0.1 \pm 0.01$ & $49 \pm 3$ & $76 \pm 3$ & $0.01,-0.02$ & $350_{-12}^{+13}$ \\
ALMA 260 & $0.1 \pm 0.01$ & $49 \pm 3$ & $76 \pm 6$ & $0.004,-0.02$ & $302_{-16}^{+17}$ \\
\hline
\end{tabular}

Notes. The stated uncertainties are the $1 \sigma$ deviation from the mean of the last 1000 steps of the MCMC fit with walkers whose acceptance fraction is $>20 \%$ (see text).

$3-2, \mathrm{~N}_{2} \mathrm{D}^{+} 3-2, \mathrm{HCN} 3-2, \mathrm{HCO}^{+} 3-2, \mathrm{SO} 56-45, \mathrm{p}-\mathrm{H}_{2}^{18} \mathrm{O}$ $31,3-2_{2,0}$, and Appendix A), only a few are strongly detected in emission. While $\mathrm{N}_{2} \mathrm{D}^{+}$can be identified spectrally, its emission cannot be imaged with the current baselines coverage, suggesting that it is present in the diffuse large-scale envelope (e.g., Crapsi et al. 2005; Tobin et al. 2013). The channel maps and zeroth moment maps of the strong lines are shown in Figs. 4 and 5.

In the inner $1^{\prime \prime}$ square aperture, ${ }^{12} \mathrm{CO}$ line emission is the strongest with a peak of $0.5 \mathrm{Jy}$. The peak line flux densities of ${ }^{13} \mathrm{CO}, \mathrm{HCO}^{+}$, and $\mathrm{SO}$ are similar with a maximum of $\sim 0.1 \mathrm{Jy}$. The weaker lines are $\mathrm{C}^{18} \mathrm{O}$ and $\mathrm{HCN}$. The integrated flux densities are listed in Table 2 including the upper limits for a few molecular lines of interest. The tabulated integrated flux densities are calculated considering pixels whose intensities $>3 \sigma$ over the entire image. The upper limits are calculated using a spatial mask $(x, y)$ over the dust continuum emission following the methods outlined in Carney et al. (2019) assuming a Gaussian linewidth of $1 \mathrm{~km} \mathrm{~s}^{-1}$ (see Harsono et al. 2020). We also report upper limits to the rotational transitions in the vibrational bending mode of $\mathrm{HCN}\left(v_{2}=1\right)$. Their spectra are shown in Appendix B. The bending mode of HCN should be detectable toward the inner regions of protoplanetary disks in order to constrain the structure of the inner hot disk $\left(T_{\text {gas }}>500 \mathrm{~K}\right.$, Bruderer et al. 2015). The non-detection of these hot HCN lines limits the existence of hot gas to the inner $10 \mathrm{au}$ of TMC1A.

The spectral line profile of $\mathrm{C}^{18} \mathrm{O} 2-1$ is symmetric about the systemic velocity as would be expected from a rotating structure. A similar line profile is seen for ${ }^{13} \mathrm{CO} 2-1$, while the ${ }^{12} \mathrm{CO} 2-1$ line shows a stronger blue-shifted emission reflecting the presence of the extended disk wind (Bjerkeli et al. 2016). The $\mathrm{HCO}^{+}$, SO, and $\mathrm{HCN}$ molecular lines exhibit narrow line profiles between 2 and $12 \mathrm{~km} \mathrm{~s}^{-1}$ similar to $\mathrm{C}^{18} \mathrm{O}$ and ${ }^{13} \mathrm{CO}$. The HCN $J=3-2$ line has 6 hyperfine components (Mullins et al. 2016) that could be responsible for the narrow absorption features (1-2 channels wide) seen in the spectrum at velocities significantly offset from systemic. Despite the weak HCN line profile, the channel maps in Fig. 4 clearly indicate that its emission is detected in multiple channels.

The channel maps in Fig. 4 show that most of these molecular lines are detected in the vicinity of the millimeter dust continuum continuum emission. ${ }^{12} \mathrm{CO},{ }^{13} \mathrm{CO}, \mathrm{C}^{18} \mathrm{O}$, and $\mathrm{HCO}^{+}$ show strong molecular emission from $1-12 \mathrm{~km} \mathrm{~s}^{-1}$ while both $\mathrm{HCN}$ and SO are detected between 2.5 and $10 \mathrm{~km} \mathrm{~s}^{-1}$. The $\mathrm{HCO}^{+}$ and $\mathrm{C}^{18} \mathrm{O}$ show emissions that are co-spatial. The channel maps also show extended arc features in both $\mathrm{HCO}^{+}$and $\mathrm{HCN}$ lines that are due to filtered-out emission (Tychoniec et al. in prep.). The zeroth moment maps (Fig. 5) show clearly these arcs. The observed molecular lines avoid the central 30 au radius due to the optically thick dust and forms a ring-like structure (Harsono et al. 2018). Meanwhile, the integrated SO emission is in a shape of a ring that extends up to 0.'5 away, which has been observed toward other protostars (e.g., Sakai et al. 2014b).

The observed HCN emission peaks at two locations that are marked by pink and orange boxes in Fig. 5. In order to investigate the origin of these $\mathrm{HCN}$ peaks, we extract an average spectrum over the two regions. Figure 6 shows the spectrum of $\mathrm{HCN}$ compared to ${ }^{12} \mathrm{CO}$ and $\mathrm{C}^{18} \mathrm{O}$ in the region to the north of the disk (pink box). The broad ${ }^{12} \mathrm{CO}$ emission indicates a molecular emission from the outflow cavity wall (with a peak at $\approx 2.5 \mathrm{~km} \mathrm{~s}^{-1}$; Bjerkeli et al. 2016) and a faster outflow/molecular wind component at $\approx-3 \mathrm{~km} \mathrm{~s}^{-1}\left(\approx v_{\text {sys }}-9 \mathrm{~km} \mathrm{~s}^{-1}\right)$. The narrow peak of $\mathrm{HCN}$ emission (at $\approx 5 \mathrm{~km} \mathrm{~s}^{-1}$ ) is similar to that of $\mathrm{C}^{18} \mathrm{O}$. The other region we highlight is located to the west (orange box). Figure 7 shows the comparison between $\mathrm{HCN}_{1} \mathrm{HCO}^{+}, \mathrm{SO}$, and $\mathrm{C}^{18} \mathrm{O}$ spectra in this region. The similarity in their line profiles are indicating a common origin.

In an attempt to extract the DCN emission, a proxy mask is created from the $\mathrm{C}^{18} \mathrm{O}$ spectral cube by taking pixels $(x, y, v)$ that are $>3 \sigma$. Figure 8 demonstrates that it can extract the $\mathrm{HCN}$ emission corresponding to the red-shifted Keplerian disk at $v \sim 9 \mathrm{~km} \mathrm{~s}^{-1}$ ) as evidenced by its similarity to the $\mathrm{C}^{18} \mathrm{O}$ spectra. The DCN emission is, however, still not clearly detected over the same regions that $\mathrm{C}^{18} \mathrm{O}$ is observed.

\section{Modeling results: molecular column densities and excitation conditions}

\subsection{Molecular column density of the disk}

The molecular abundance structure of the TMC1A disk can be derived from the spatially resolved molecular column densities. To zeroth order, the molecular column density of the emitting gas can be determined by assuming a thermalized, optically thin layer of emission (Goldsmith \& Langer 1999; Mangum \& Shirley 2015). As an example, we first present the detailed 

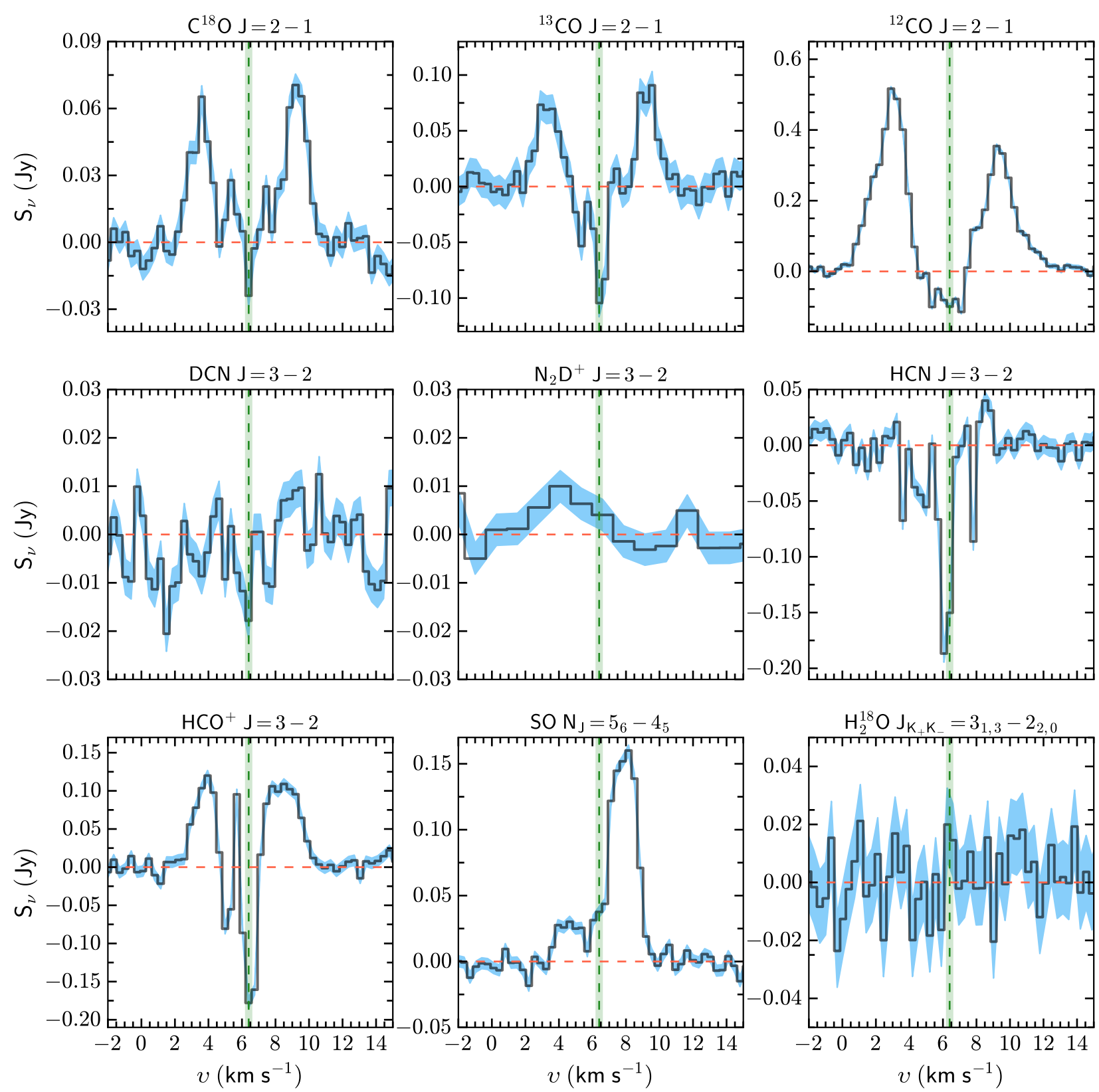

Fig. 3. Observed molecular spectra from the inner 1" square region centered on TMC1A. The green vertical line is the systemic velocity of the system, while the horizontal, red dashed line indicates the baseline. The $1 \sigma$ error is shown by the shaded blue regions. See Table 2 for more information on each line.

analysis of the $\mathrm{C}^{18} \mathrm{O}$ emission and compare the results from the optically thin approximation to a non-LTE radiative transfer analysis of a slab. The same analysis is applied to the other molecular lines.

The integrated strength of the $\mathrm{C}^{18} \mathrm{O}$ emission across the map ranges from 6 to $15 \mathrm{mJy} \mathrm{km} \mathrm{s}^{-1}$ per beam, which translates to $12-30 \mathrm{~K} \mathrm{~km} \mathrm{~s}^{-1}$. In the optically thin approximation, adopting an excitation temperature of $30 \mathrm{~K}$, the integrated column density of $\mathrm{C}^{18} \mathrm{O}$ along the line of sight is then $\approx 10^{16} \mathrm{~cm}^{-2}(\tau \approx 1)$. A temperature of $30 \mathrm{~K}$ is usually adopted to convert the dust thermal emission to dust mass and, in the case of $\mathrm{C}^{18} \mathrm{O}$ at least, most of the molecular emission is emitted from a region where $T_{\text {gas }}=T_{\text {dust }}$ (e.g., Ceccarelli et al. 1996; Doty et al. 2002). A non-LTE radiative transfer calculation of a slab using RADEX (van der Tak et al. 2007) is also performed as a check on the column density and excitation temperature. These models predict a kinetic temperature of $\mathrm{C}^{18} \mathrm{O}$ between 5 and $30 \mathrm{~K}$ with $\tau=0.1-1$. The $N_{\mathrm{C}^{18} \mathrm{O}}$ is between $10^{15}$ and $10^{16} \mathrm{~cm}^{-2}$. Therefore, the optically thin limit provides a reasonable estimate for the $\mathrm{C}^{18} \mathrm{O}$ emitting layer.

Since the $\mathrm{C}^{18} \mathrm{O}$ emission can be marginally optically thick $(\tau \approx 1)$, line emission from the other observed CO isotopologs will definitely be optically thick $(\tau \approx 10)$ simply from the isotopic ratios. Therefore, we present the non-LTE calculations here, and adopt the CO collisional rate coefficients from Yang et al. (2010), obtained from the Leiden Atomic and Molecular Database (LAMDA; Schöier et al. 2005). These rates coefficients consider two collisional partners of ortho- and para- $\mathrm{H}_{2}$ (Jankowski \& Szalewicz 2005). The integrated line intensity of ${ }^{13} \mathrm{CO}$ is between 16 and $55 \mathrm{~K} \mathrm{~km} \mathrm{~s}^{-1}$ while it is $30-300 \mathrm{~K} \mathrm{~km} \mathrm{~s}^{-1}$ for ${ }^{12} \mathrm{CO}$. The non-LTE radiative transfer model yields a suitable range of the excitation temperatures in between 15 and $\sim 70 \mathrm{~K}$ to 


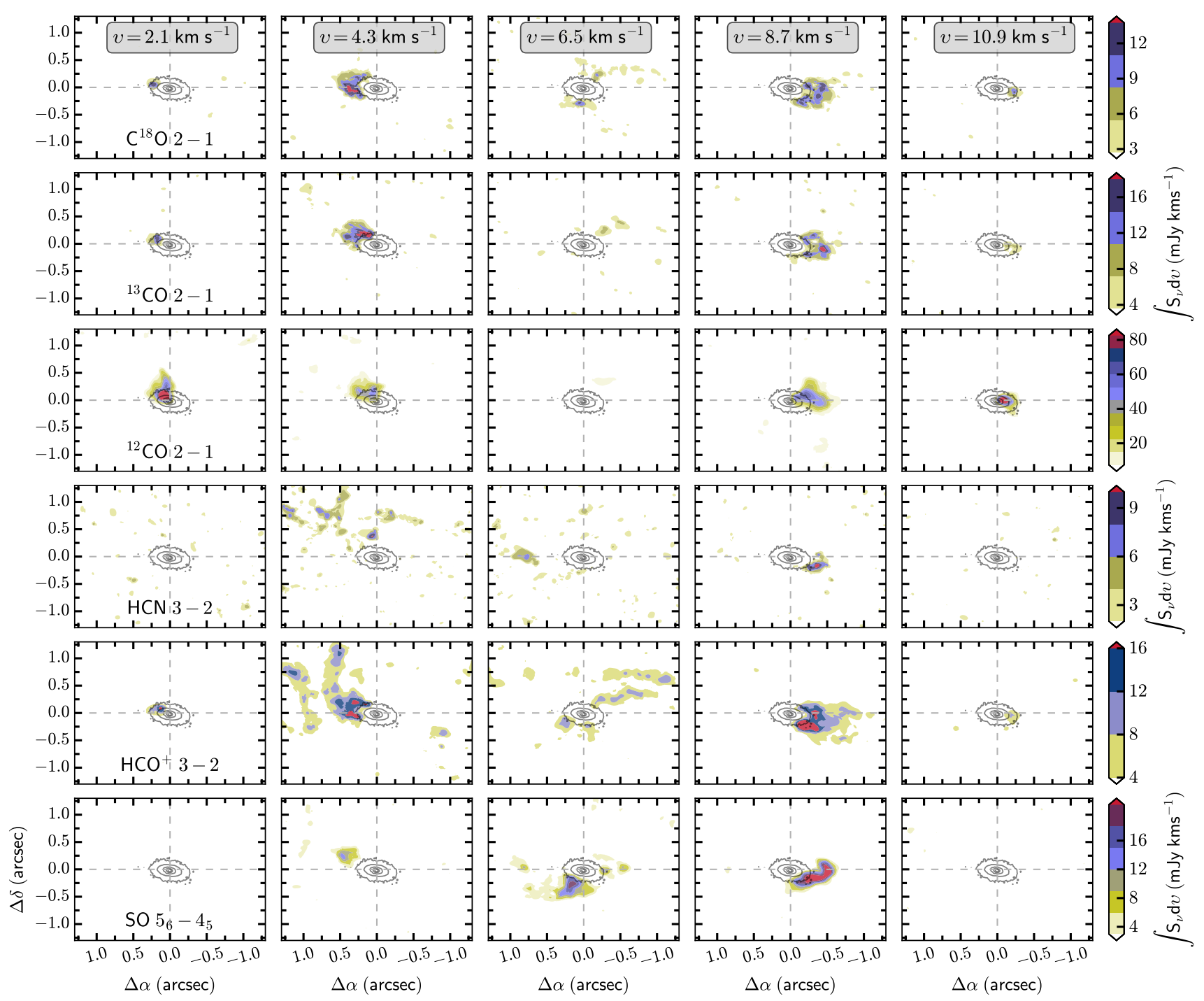

Fig. 4. Channel maps of the six brightest molecular lines are shown: $\mathrm{C}^{18} \mathrm{O} 2-1,{ }^{13} \mathrm{CO} 2-1,{ }^{12} \mathrm{CO} 2-1, \mathrm{HCN} 3-2$, $\mathrm{HCO}^{+} 3-2$, and $\mathrm{SO} 5{ }_{6}-45$ (top to bottom). Each panel shows the line intensities integrated over six channels (left to right) and after clipping pixels with intensities $<3 \sigma$. The grey box in the first row indicates the average velocity of the six channels. The color scale for molecular line is shown at the right hand side. The dust continuum emission of the aggregated observations is plotted linearly as black contours from $5 \sigma$ to maximum intensity. Beam sizes for each line can be found in Table 2.

reproduce the observed integrated ${ }^{13} \mathrm{CO}$ emission with column densities between $10^{16}$ and $10^{17} \mathrm{~cm}^{-2}$. In the case of ${ }^{12} \mathrm{CO}$ the range of kinetic temperature is $50-300 \mathrm{~K}$ and ${ }^{12} \mathrm{CO}$ column densities between $10^{17}$ and $10^{19} \mathrm{~cm}^{-2}(\tau=1-7)$. Since both ${ }^{13} \mathrm{CO}$ and ${ }^{12} \mathrm{CO}$ lines are optically thick, the observed line emission comes from a surface layer (a slab) above the midplane. This implies that the derived column densities are lower limits to the total column density of the gas that is present in the system.

The non-LTE radiative transfer calculations for $\mathrm{HCN}, \mathrm{HCO}^{+}$, and SO lines also use the LAMDA collisional rate coefficients. The rates for HCN from LAMDA are based on $\mathrm{HCN}-\mathrm{He}$ collisions of Green \& Thaddeus (1974), scaled to $\mathrm{H}_{2}$. The collisional rates for $\mathrm{HCO}^{+}$are estimated using the dipole moment of Botschwina et al. (1993). SO- $\mathrm{H}_{2}$ collisional rates are scaled from SO-He calculations of Lique et al. (2006). All of these rates consider a single collisional partner, $\mathrm{H}_{2}$, without taking into account its ortho-to-para ratio.

In the optically thin limit, the observed $\mathrm{HCN}, \mathrm{HCO}^{+}$, and SO emissions are produced by $N_{\mathrm{HCN}}=5 \times 10^{12}-10^{13} \mathrm{~cm}^{-2}$, $N_{\mathrm{HCO}^{+}}=5 \times 10^{12}-10^{13} \mathrm{~cm}^{-2}$, and $N_{\mathrm{SO}}=10^{14}-6 \times 10^{14} \mathrm{~cm}^{-2}$, respectively, with an adopted excitation temperature of $30 \mathrm{~K}$. Figure 9 shows the azimuthally averaged integrated line intensities and associated column densities in the optically thin limit (including ${ }^{12} \mathrm{CO}$ and ${ }^{13} \mathrm{CO}$ ). With the non-LTE slab model, the observed $\mathrm{HCO}^{+}$emission can be reproduced by kinetic temperatures between 30 and $50 \mathrm{~K}$ and column densities of $10^{13} \mathrm{~cm}^{-2}$, which are similar to the derived values presented in Fig. 9 $(\tau \approx 1)$. Similar temperatures can also reproduce the observed $\mathrm{HCN}$ emission with $N_{\mathrm{HCN}} \approx 10^{12}-10^{13} \mathrm{~cm}^{-2}, \tau<1$. Finally, the observed SO emission can be reproduced with similar kinetic temperatures as the previous two molecules and higher column densities of $10^{14}-10^{15} \mathrm{~cm}^{-2}$. The physical conditions of SO along the line of sight are consistent with ${ }^{13} \mathrm{CO}$, which is optically thick. The optically thin LTE calculations can provide useful limits to the column densities of the detected molecules.

Since we have observed the same rotational transition in HCN and DCN, albeit only an upper limit on the DCN emission, we can place constraints on the $\mathrm{D} / \mathrm{H}$ ratio in the vicinity of TMC1A. We restrict this analysis to the disk-averaged value by determining the column density and temperature of the upper 



Fig. 5. Zeroth moment maps of the six most strongly detected molecular lines. They are calculated by integrating the emission from 1 to $12 \mathrm{~km} \mathrm{~s}^{-1}$, considering only pixels where the emission $>3 \sigma(1 \sigma$ values are listed in Table 2$)$. The synthesized beams are indicated in the lower left. The dust continuum emission of the aggregated observations is plotted linearly as black contours from $5 \sigma$ to maximum intensity. There are two highlighted regions in the integrated HCN map, and one in the SO map: the supposed outflow component is shown in pink and the supposed disk component is in orange. See the text for details.



Fig. 6. $\mathrm{HCN},{ }^{12} \mathrm{CO}$, and $\mathrm{C}^{18} \mathrm{O}$ spectra from the region north of the disk, indicated by the pink rectangle in Fig. 5. The -3 to $3 \sigma$ levels for the $\mathrm{HCN}$ emission is indicated by the shaded region centered at $0 \mathrm{mJy}$ beam ${ }^{-1}$. The outflow component seen in ${ }^{12} \mathrm{CO}$ is annotated. The green vertical line denotes the systemic velocity.

limit to the integrated line intensity obtained from the $\mathrm{HCN}$ and DCN spectra, summed over the Keplerian disk. As a check, we determined that the physical conditions derived from the averaged HCN spectrum are similar to the azimuthally-averaged values in the previous section. For a range of excitation temperatures between 15 and $50 \mathrm{~K}$, an average value of $N_{\mathrm{HCN}} \sim$ $1.1 \times 10^{12} \mathrm{~cm}^{-2}$ is derived for the TMC1A disk. The same analysis provides an upper limit for the DCN column density

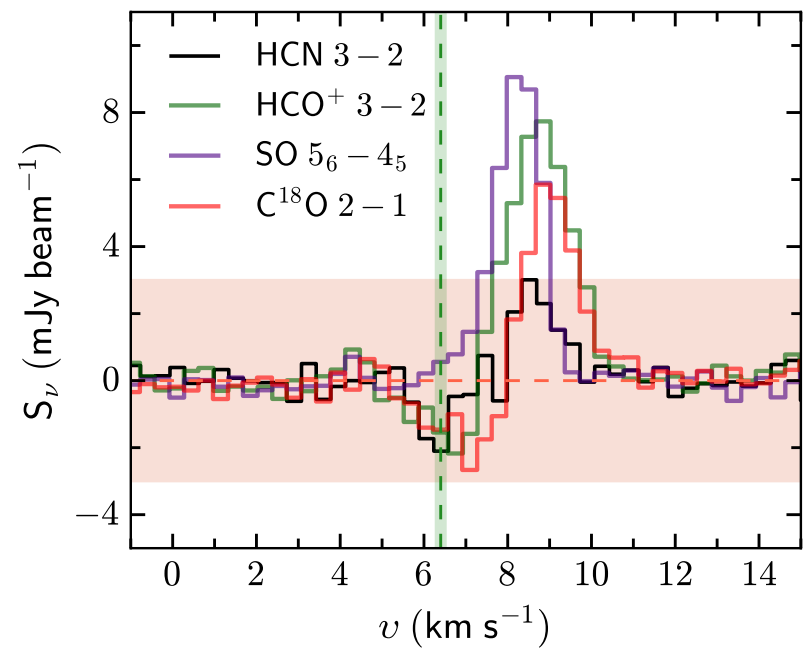

Fig. 7. $\mathrm{HCN}, \mathrm{HCO}^{+}$, $\mathrm{SO}$, and $\mathrm{C}^{18} \mathrm{O}$ spectra from the western part of the disk (denoted by the orange rectangle in Fig. 5). The shaded region highlights the -3 to $3 \sigma$ levels of the $\mathrm{HCN}$ emission, while the red horizontal dashed line shows the baseline, and the green vertical line shows the systemic velocity.

of $\sim 3 \times 10^{11} \mathrm{~cm}^{-2}$, resulting in a DCN/HCN ratio of $<0.3$. If we instead only consider the HCN emission between 8 and $10 \mathrm{~km} \mathrm{~s}^{-1}$ (where the HCN emission is strongest), the upper limit of $\mathrm{DCN} / \mathrm{HCN}$ is then $<0.26$.

\subsection{Temperature structure of the disk}

Spatially resolved observations of dust continuum and molecular line emission can be used to estimate the temperature structure 

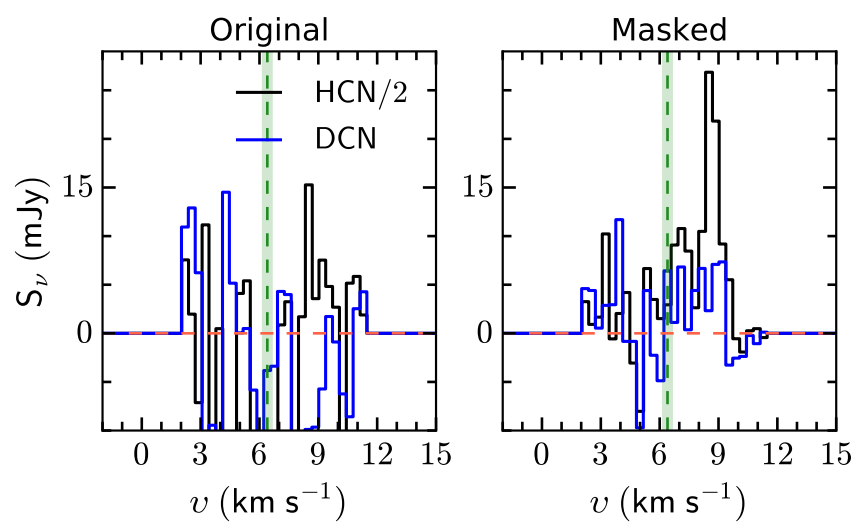

Fig. 8. Comparison between the original HCN (black) and DCN (blue) spectra with the masked spectra. The original spectra are the same as Fig. 3. The mask is created from the $\mathrm{C}^{18} \mathrm{O}$ channel maps (see text) to highlight the molecular emission associated with the Keplerian disk.
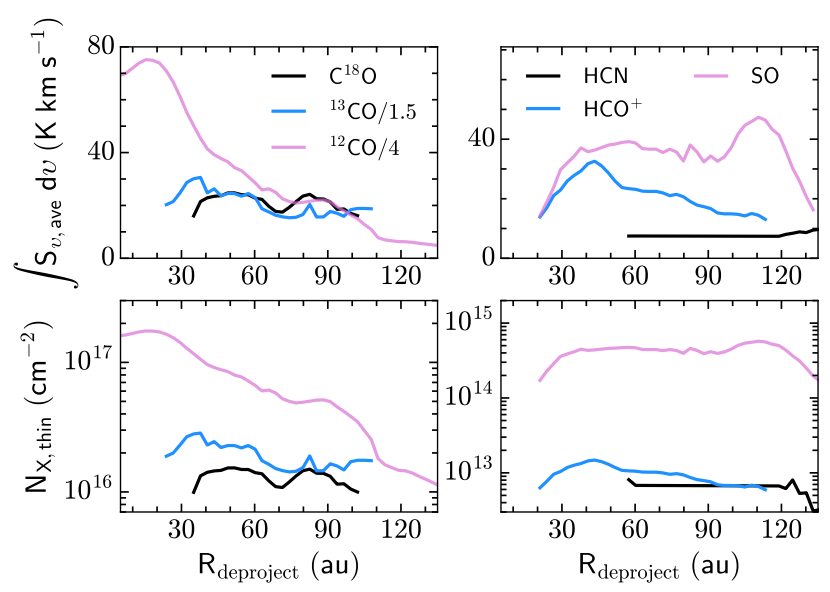

Fig. 9. Azimuthally averaged, integrated line intensities (top) and column densities (bottom) as a function of the deprojected radius. The column densities are derived assuming optically thin molecular line emission, and an excitation temperature of $30 \mathrm{~K}$. The deprojection utilizes the $i$ and PA obtained in Sect. 3.1.

of the disk. To this end, Fig. 10 shows the observed dust continuum brightness temperature at 230 and $240 \mathrm{GHz}$, as well as the molecular line peak brightness temperatures, as a function of the deprojected radius. The flattening in the inner $<10$ au is caused by unresolved continuum emission. If the dust emission is optically thick within the inner $30 \mathrm{au}$, however, the peak continuum brightness temperature gives indications on the disk's temperature profile.

Using the high-velocity channels $\left(1<v<4 \mathrm{~km} \mathrm{~s}^{-1}\right.$ and $9<v<12 \mathrm{~km} \mathrm{~s}^{-1}$; to avoid resolved-out emission near the systemic velocity), the peak brightness temperature of ${ }^{13} \mathrm{CO}, \mathrm{HCO}^{+}$, $\mathrm{HCN}$ and SO molecular lines as a function of the deprojected radius is shown in Fig. 10. There are only small differences between the ${ }^{13} \mathrm{CO}$ and $\mathrm{C}^{18} \mathrm{O}$ brightness temperatures, and only at $>30$ au radius, so only ${ }^{13} \mathrm{CO}$ is plotted. After acknowledging the decrease in molecular emission in the inner 30 au radius, as well as optically thin components, the remaining few optically thick emission data points can provide the additional constraints on the disk temperature profile. Their approximate average values and locations are indicated by the large symbols in Fig. 10.

The equilibrium midplane temperature of a circumstellar disk can be characterized using a power-law in radius with

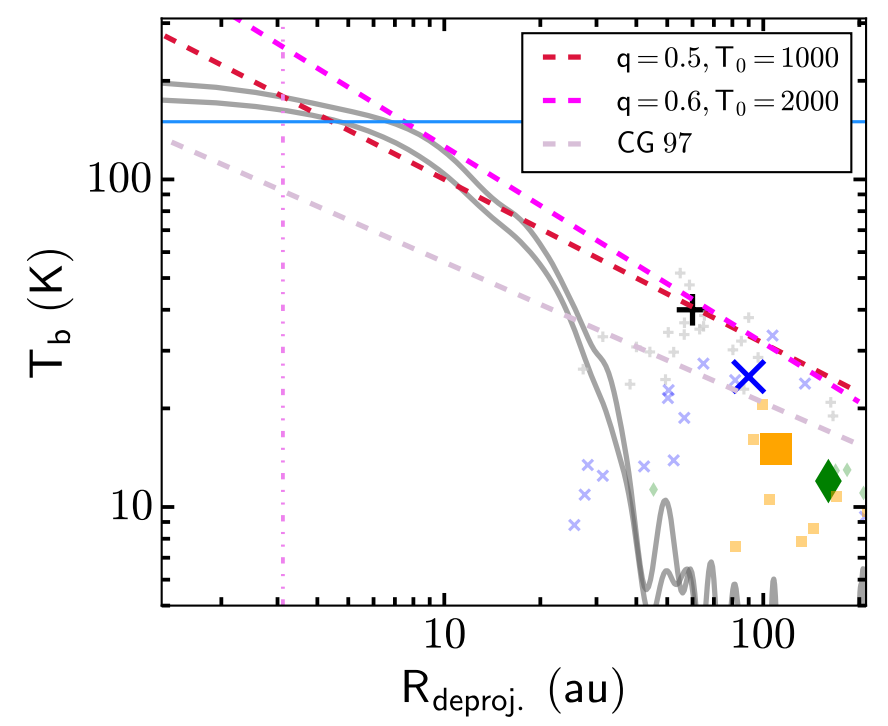

Fig. 10. Brightness temperature profile along the disk major axis as a function of deprojected radius. Gray solid lines show the observed dust continuum brightness profile at 230 and $240 \mathrm{GHz}$. Peak brightness temperatures of ${ }^{13} \mathrm{CO}$ (black + ), $\mathrm{HCO}^{+}$(blue crosses), $\mathrm{HCN}$ (green diamonds), and SO (orange squares) are also plotted. The smaller symbols show the observed peak temperatures at velocities between 1 and $4 \mathrm{~km} \mathrm{~s}^{-1}$ and 9-12 $\mathrm{km} \mathrm{s}^{-1}$ that contain $>3 \sigma$ emission, while the large symbols indicate the averaged values of these points. For comparison, a power-law $\left(T=T_{0}(R / 0.1 \mathrm{au})^{q}\right)$ and the Chiang \& Goldreich(1997; CG97) temperature profiles are also shown (dashed lines). The values of $T_{0}$ and $q$ are indicated in the legend. The vertical purple dashed-dot line indicates $0.5 \times$ the beam size, while the horizontal blue line denotes the water snowline at $160 \mathrm{~K}$ (Meijerink et al. 2009).

an index between -0.4 and -0.5 (Chiang \& Goldreich 1997; van 't Hoff et al. 2018b). We find that, by eye, a temperature profile given by $2000 \times(R / 0.1 \mathrm{au})^{-0.6}$ seems to be consistent with our dust and gas observations of TMC1A. It reproduces the dust brightness temperature profile in the inner 30 au and intersects the peak of the ${ }^{13} \mathrm{CO}$ emission. The derived temperature structure is similar to the disk around $\operatorname{L1527}\left(L_{\mathrm{bol}}=1.9-2.6 L_{\odot}\right)$ as measured by van 't Hoff et al. (2018b). Furthermore, the slope of the temperature profile is steeper than expected from purely re-radiated stellar photons $(q \simeq-0.4)$, implying that the observed emitting surface shifts from the hot disk surface to the cold disk/envelope gas at large radii. The estimated temperature of $1000-2000 \mathrm{~K}$ at $0.1 \mathrm{au}$ is also consistent with the observed $L_{\text {bol }}\left(2.7 L_{\odot}\right)$ for a $4000 \mathrm{~K}$ protostar $\left(R_{\star} \sim 3.4 R_{\odot}\right)$. The derived temperature structure implies that the warm TMC1A disk does not have a $\mathrm{CO}$ freeze-out region $\left(T_{\text {dust }}=30 \mathrm{~K}\right)$ in the inner 100 au of the disk (van 't Hoff et al. 2018b). Recent $C^{17} \mathrm{O}$ observations presented by van 't Hoff et al. (2020) also indicate the lack of $\mathrm{CO}$ freeze-out region in TMC1A disk.

\section{Discussion: the molecular layer of disks}

The unprecedented spatial resolution of these observations allows us to disentangle molecular emission from the disk and from the large-scale envelope. The simple molecules targeted in our data set are ${ }^{12} \mathrm{CO},{ }^{13} \mathrm{CO}, \mathrm{C}^{18} \mathrm{O}, \mathrm{SO}, \mathrm{HCO}^{+}, \mathrm{HCN}$, and DCN, plus the spectrally unresolved $\mathrm{N}_{2} \mathrm{D}^{+}$. These molecules can be used to better understand the evolution of the physical and chemical structure of disks during the embedded stage of star and planet formation. 




Fig. 11. A schematic of the observed molecular emission and the physical components of the embedded protostellar system TMC1A. For the references given in the illustration, 'et al.' is abbreviated as “+”. References: Bjerkeli et al. (2016), Harsono et al. (2018), van 't Hoff et al. (2020). Main panel: molecular gas components projected in the same way as in the observation. Inset at bottom right: interpretation of relative location of the origin of molecular gas emission shown in the $(x, z)$ plane, with $z$ along the rotation axis of the disk.

\subsection{Physical components of embedded protostars traced by molecular lines}

The observed molecular lines trace different physical components of an embedded protostellar system, i.e., the inner envelope ( $R<500 \mathrm{au}$ ), Keplerian disk, disk wind, and the cold, infalling envelope. The schematic in Fig. 11 summarizes the observed molecular tracers, their emitting regions, and the physical component that we associate each tracer to. As done previously, we use the symmetric line profile of ${ }^{13} \mathrm{CO}$ and $\mathrm{C}^{18} \mathrm{O} 2-1$ to establish the location of the Keplerian disk. We then compare the other molecular line profiles with ${ }^{13} \mathrm{CO}$ and $\mathrm{C}^{18} \mathrm{O}$ to provide a first look into the physical components traced by these other lines in TMC1A. Finally, we add the channel maps and zeroth moment maps comparison to give the complete understanding on the emitting regions of these molecules and the structure of the TMC1A system.

From the line profiles in Fig. 3, the roughly symmetric $\mathrm{HCO}^{+}$ 3-2 line profile indicates that it is tracing the Keplerian disk. This is not unexpected since $\mathrm{HCO}^{+}$is mainly formed through reactions that involve $\mathrm{CO}$ (see Sect. 5.3), and, thus, $\mathrm{HCO}^{+}$emission should coincide with the $\mathrm{CO}$ emission. On the surface of the disk with low densities, chemical reactions tend to favor the production of $\mathrm{N}_{2} \mathrm{H}^{+}$(Aikawa et al. 2015) and the destruction of $\mathrm{HCO}^{+}$by water vapor (Jørgensen et al. 2013; van 't Hoff et al. 2018a). The emitting $\mathrm{HCO}^{+}$layer in the disk is confined to a small outer layer (see Mathews et al. 2013).

The asymmetric line profiles of $\mathrm{HCN}$ and SO, meanwhile, strongly indicate that these lines are tracing multiple physical components along the line of sight (Fig. 7). For example, both $\mathrm{HCN}$ and SO show red-shifted emission to the west that corresponds (both spatially and in velocity space) to the Keplerian disk as traced by $\mathrm{C}^{18} \mathrm{O}$ and $\mathrm{HCO}^{+}$.

The narrow line profile of HCN 3-2 (Figs. 6 and 7) strongly indicates emission from quiescent gas, and this gas could be in either the envelope or Keplerian disk. The similarity in the line shape and velocity peak of $\mathrm{HCO}^{+}$and $\mathrm{C}^{18} \mathrm{O}$ in Fig. 7 points to the Keplerian disk as the origin of the red-shifted $\mathrm{HCN}$ emission. At lower velocities, the HCN line profiles shows evidence of some contribution from the surrounding envelope. Therefore, we conclude that the HCN line traces the disk-envelope transition region. A detailed 2D physical and chemical model is required to quantify the exact location of the molecular emission, but this is beyond the scope of the current work. Finally, the morphology and velocity of HCN 3-2 emission (Figs. 5, 6) does not appear to be connected to the outflow observed previously in $\mathrm{CO}$ (Bjerkeli et al. 2016).

Our spatially resolved SO observations show morphological features (Figs. 4 and 5) that are similar to $\mathrm{CO}$ and $\mathrm{HCO}^{+}$. The narrow line profile of SO in Fig. 7 and the low peak temperatures of SO in Fig. 10 meanwhile rule out an accretion shock origin (e.g., Sakai et al. 2014b). A comparison between the zeroth moment maps of $\mathrm{C}^{18} \mathrm{O}, \mathrm{HCN}$, and ${ }^{12} \mathrm{CO}$ is shown in Fig. 12. Since the SO and ${ }^{12} \mathrm{CO}$ molecular emission are not co-spatial, it excludes a disk wind origin for SO (e.g. Tabone et al. 2017) for TMC1A. However, the blue-shifted HCN emission in Fig. 12 peaks at the location near the blue-shifted $\mathrm{CO}$ emission suggesting a region where the wind and the envelope interact. The 


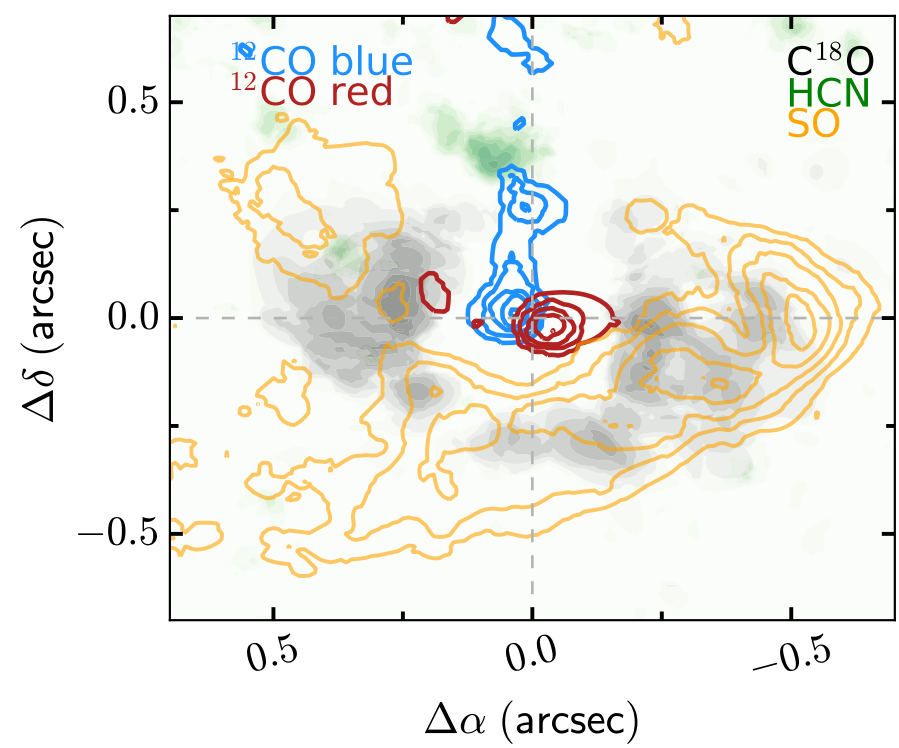

Fig. 12. Comparison of $\mathrm{SO}, \mathrm{C}^{18} \mathrm{O}, \mathrm{HCN}$, and ${ }^{12} \mathrm{CO}$ emitting regions using zeroth moment maps. The $\mathrm{C}^{18} \mathrm{O}$ emission is shown in grayscale with a linear scaling between 0.2 and 0.8 of the maximum in order to highlight the disk component. Green filled contours shows $\mathrm{HCN}$ integrated from -1 to $6 \mathrm{~km} \mathrm{~s}^{-1}$. The color spans linearly from 0.3 to 1.0 of the maximum value. Blue-shifted ${ }^{12} \mathrm{CO}\left(-7\right.$ to $\left.-1 \mathrm{~km} \mathrm{~s}^{-1}\right)$ and redshifted $\left(13-19 \mathrm{~km} \mathrm{~s}^{-1}\right)$ emission are highlighted by the blue and red contours, respectively. The contours span linearly from 0.15 to 1.0 of the maximum. Finally, the SO integrated emission is shown using orange contours with a linear scaling from 0.15 to 1.0 of the maximum.

combination of the SO line profile and its peak brightness temperature (Fig. 10) indicates that it originates from the infalling, warm inner envelope along the line of sight. The location of the $\mathrm{SO}$ emission with respect to the disk wind (blue-shifted emission at $4.3 \mathrm{~km} \mathrm{~s}^{-1}$ ) and the Keplerian disk further supports that SO is located along the outflow cavity wall. This is also hinted by the presence of lower velocity component in the spectrum shown in Fig. 7. The favorable orientation of TMC1A and these deep spectrally resolved molecular line observations allow us to disentangle the different physical components of this embedded disk. Such an empirical derivation of the origin of SO would have been impossible in an edge-on system and without additional gas line tracers.

\subsection{Molecular abundances in the TMC1A disk}

One of the major questions in star and planet formation is whether or not planet-forming materials enter the disk from the envelope unaltered. Alteration of planet-forming materials require high temperatures that can generally only be achieved in interstellar shocks and the inner regions of protostellar systems. These changes would be relevant for the accretion history of the protostar (e.g., dust sublimation in the inner regions of the disk or protostellar accretion bursts) and disk formation (e.g., accretion shocks at the disk-envelope interface that affect the chemistry and ionization fraction) that can be explored by comparing the chemical abundances of young stellar objects at different scales. Indeed, differences in chemical content between the embedded disk and its surrounding envelope may point to on-going physical processes during the early stages of star and planet formation.

The molecular line spectra and the kinetic temperature analysis presented in this work identify some regions and velocity intervals that emission from the disk, but also indicate that some lines of sight toward TMC1A are contaminated by its surrounding envelope. We showed in Sect. 4.1 that assuming the optically thin limit can provide useful constraints on the emitting molecular layer and the column densities of the disk. Furthermore, with the azimuthally averaged column densities in hand, we can constrain the molecular abundances in the disk. In addition, since these observations use the longest baselines configuration of ALMA, they are only sensitive up to a maximum recoverable scale of $\sim 0$ '. 5 ( $\sim 70 \mathrm{au})$. Thus, the observed molecular emission is restricted to the inner hundred au of TMC1A, however, the chemical timescales in these regions are indeed longer than the infall timescale (the time for the gas to fall onto the disk). Therefore, we expect that the derived molecular abundances on the observed scales of these observations should not significantly change before the molecular gas enters the disk.

By comparing the derived column densities of the different species, we obtain $N_{\mathrm{HCO}^{+}} / N_{{ }^{13} \mathrm{CO}} \sim 10^{-4}-10^{-3}$ and $N_{\mathrm{SO}} / N_{{ }^{13} \mathrm{CO}}=$ $10^{-3}-10^{-1}$. The HCN abundance is meanwhile estimated through comparison with the $\mathrm{C}^{18} \mathrm{O}$ column density, giving $N_{\mathrm{HCN}} / N_{\mathrm{C}^{18} \mathrm{O}}=10^{-4}-10^{-2}$. The proxy mask (see Sect. 3.2) using the $\mathrm{C}^{18} \mathrm{O}$ spectral cube recovers more $\mathrm{HCN}$ flux than a proxy mask using the ${ }^{13} \mathrm{CO}$ spectral cube. Although some envelope material is contaminating the detected emission, it is still useful to calculate the relative abundances for the disk, which are shown graphically in Fig. 13. Adopting the ISM isotopic ratios of ${ }^{12} \mathrm{C}:{ }^{13} \mathrm{C}=70$ and ${ }^{16} \mathrm{O}:{ }^{18} \mathrm{O}=540$ (Wilson \& Rood 1994), the derived molecular abundances relative to ${ }^{12} \mathrm{CO}$ are then $X_{\mathrm{HCO}^{+}} / X_{\mathrm{CO}} \sim 10^{-6}-10^{-5}, X_{\mathrm{HCN}} / X_{\mathrm{CO}} \sim 10^{-7}-10^{-5}$, and $X_{\mathrm{SO}} / X_{\mathrm{CO}} \sim 10^{-5}-10^{-3}$.

It has been demonstrated that the $\mathrm{CO}$ gas abundance in young disks remains close to the canonical ISM value of $X_{\mathrm{CO}}=10^{-4}$ relative to $\mathrm{H}_{2}$ (Harsono et al. 2014; van 't Hoff et al. 2018b; Zhang et al. 2020). Adopting this value, we estimate abundances of $X_{\mathrm{HCO}^{+}}=10^{-10}-10^{-9}, X_{\mathrm{HCN}}=10^{-11}-10^{-9}$ and $X_{\mathrm{SO}}=10^{-9}$ $10^{-7}$ in the disk of TMC1A, where $X$ denotes an abundance with respect to $\mathrm{H}_{2}$. One should keep in mind, however, that these abundance fractions are valid in the emitting regions of the inner warm envelope and the layers of the young disk above the dust photosphere.

Remarkably, the gas abundance ratios in the disk around TMC1A are similar to its protostellar envelope (Fig. 13). This implies a smooth transport of materials without the presence of strong shocks $(T>100 \mathrm{~K})$ that modify the molecular composition of the material en route to the disk (Visser et al. 2009). $\mathrm{HCO}^{+}$and $\mathrm{HCN}$ show features of filtered out molecular emission. The comparison of the total $\mathrm{C}^{18} \mathrm{O}$ and ${ }^{13} \mathrm{CO}$ integrated emission between this study and Harsono et al. (2014) also indicate $90 \%$ of the emission is filtered out as a result of going from 0.5 to $0 . ' 1$ resolution. However, the lack of absorption in the SO line profile suggests that our studies recover most of its emission. Thus, the range of the SO/CO abundance ratio shown in Fig. 13 may instead be explained by filtered out $\mathrm{CO}$ emission. It further indicates that $\mathrm{HCO}^{+}$and $\mathrm{SO}$ are not drastically affected by the physical processes that take place during disk formation.

The chemical composition of the TMC1A disk is also similar to that of Class II disks. The main difference is the lower absolute $\mathrm{CO}$ gas abundance that is inferred from $\mathrm{CO}$ observations of Class II disks (e.g., Miotello et al. 2017; Zhang et al. 2020). CO depletion during star and planet formation is linked to the formation of complex organics, on-going planet formation, and the UV field around low-mass stars (e.g., Yu et al. 2017; Bosman et al. 2018; Dodson-Robinson et al. 2018; McClure 2019). As such, gas abundance ratios (as compared to absolute abundances) may be less sensitive to variations between objects and better reflect how 


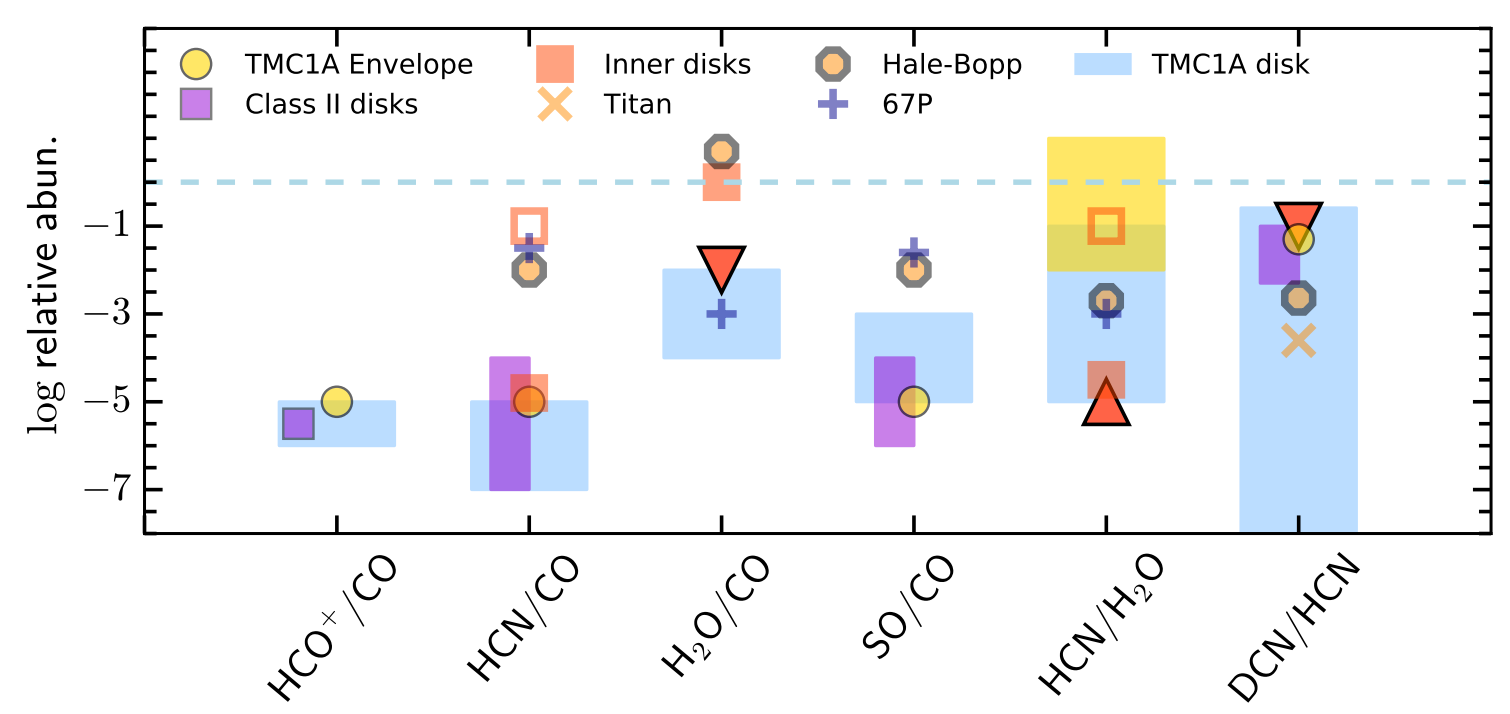

Fig. 13. Comparison of the relative molecular gas abundances in the TMC1A disk and its envelope, Class II disks, and Solar System bodies. The range of values obtained in this work is shown by the blue shaded rectangles. The red arrows indicate lower and upper limits. Yellow circles indicate the molecular abundance ratios of the protostellar envelope around TMC1A from Jørgensen et al. (2004). The abundances of HCN and DCN in the envelope are approximated to the values in Schöier et al. (2002), Roberts et al. (2002), and Tafalla et al. (2006). The water gas fraction in the protostellar envelope is assumed to be between $10^{-7}$ and $10^{-4}$ based on models by Caselli et al. (2012) and Schmalzl et al. (2014). Molecular abundances in Class II disks (purple rectangles) are based on values from Mathews et al. (2013), Teague et al. (2015), Guzmán et al. (2015), Cleeves et al. (2018), Huang et al. (2017), and Booth et al. (2019). The inner disk hot gas molecular abundances (empty red squares) are adopted from Najita et al. (2013) and Salyk et al. (2011) shown by filled red squares. The abundances of comet Hale-Bopp are based on Bockelée-Morvan et al. (2000). The abundances of comet 67P/Churyumov-Gerasimenko are adopted from Rubin et al. (2019). The DCN/HCN fraction for Titan and comet Hale-Bopp are obtained from Molter et al. (2016) and Meier et al. (1998), respectively.

chemistry changes with evolutionary state. Therefore, the similar gas molecular abundance ratios between the TMC1A disk and Class II disks may indicate that the molecular layer of protoplanetary disks do not change significantly once the disk is formed $\left(M_{\mathrm{d}} / M_{\mathrm{env}} \sim 0.5\right)$.

Interestingly, for the most part, the abundance ratios between protostellar systems and Solar System objects do show significant differences. In order to assess whether SO is truly being affected by disk formation, deep observations of other $\mathrm{S}$-bearing species (e.g., $\mathrm{H}_{2} \mathrm{~S}, \mathrm{SO}_{2}, \mathrm{CS}$ ) in combination with multi-resolution $\mathrm{CO}$ observations are needed to recover the total emission from the inner 500 au of the TMC1A system. Without observations of other S-bearing molecules, it is difficult to conclude the origin of this discrepancy. A comparison between S-bearing species in IRAS 16293-2422 and comet 67P imply that the Solar System formed in a higher UV field than IRAS 16293-2422 (Drozdovskaya et al. 2018) since $S$ is a volatile element and sensitive to the changes in the UV field. Thus, it would seem that the differences in S-bearing molecules may trace the strength of the UV field during protostellar evolution.

With regards to HCN and DCN, Huang et al. (2017) show that there is a spread in DCN abundances in disks that stems from the different cold and warm routes of DCN formation (see Aikawa et al. 1999; Willacy 2007, and references therein). Unfortunately, neither HCN nor DCN were detected toward TMC1A in the single-dish survey of Jørgensen et al. (2004) which hinders us from a straight comparison between disk and envelope values; the ratio in Fig. 13 instead adopts the prestellar core value. Our upper limits to the DCN/HCN fraction in the TMC1A disk are, meanwhile, consistent with other various young stellar objects and Solar System objects. A small variation in DCN/HCN in protostellar envelopes has been observed (Jørgensen et al. 2004), which seems to be related to the bolometric luminosity of the source. However, it is still not well understood whether the level of deuteration is modified during disk formation. For example, an older disk presented in Huang et al. (2017) seems to have a DCN/HCN ratio consistent with comet Hale-Bopp, which favors the idea of an evolving DCN/HCN ratio. Meanwhile, the DCN/HCN ratio of Titan is different than that of both disks and comets, indicating a different origin for the observed deuteration fraction.

A large variation across different types of objects is also seen in $\mathrm{HCN}$ and $\mathrm{H}_{2} \mathrm{O}$ abundance ratios. Interestingly, abundance variations in both $\mathrm{H}_{2} \mathrm{O}$ and $\mathrm{HCN}$ have been linked to angular momentum transport (Cuzzi \& Alexander 2006; Kalyaan $\&$ Desch 2019) and planet formation (e.g., Najita et al. 2013; Krijt et al. 2016; Du et al. 2017; Najita et al. 2018). Our sub-mm $\mathrm{H}_{2} \mathrm{O}$ column density for TMC1A is adopted from the upper limit in Harsono et al. (2020) $\left(N\left(\mathrm{H}_{2} \mathrm{O}\right) / N\left(\mathrm{H}_{2}\right)<10^{-6}\right)$ that probes the inner $10 \mathrm{au}$ of the disk. Hot water observations in the mid-IR around Class II disks (e.g., Carr \& Najita 2008; Salyk et al. 2011; Najita et al. 2013) meanwhile probe the inner 2 au where terrestrial planets are expected to form. The observed correlation between the $\mathrm{HCN} / \mathrm{H}_{2} \mathrm{O}$ mid-IR line flux ratios and the disk mass (via the millimeter flux density) has been suggested to trace planetesimal formation inside of 5 au (Najita et al. 2013, 2018). If the observed mid-IR line flux ratios correspond to the relative abundances in the emitting layers $(z / R \sim 0.6$, Bruderer et al. 2015), then they are consistent with the gas molecular abundance ratios derived in this work. The differences between the abundance ratios in the large-envelope and disk (Fig. 13) thus suggests that $\mathrm{HCN} / \mathrm{H}_{2} \mathrm{O}$ is set during disk formation, which is indeed supported by the similar abundance ratio observed in comets $67 \mathrm{P}$ and Hale-Bopp. On the other hand, both $\mathrm{HCN}$ and $\mathrm{H}_{2} \mathrm{O}$ abundances (relative to $\mathrm{CO}$ ) differ between the TMC1A disk, inner disks as observed in the mid-IR, and comets. However, much 
Table 4. Reaction network used to calculate the $\mathrm{HCO}^{+}$abundance given values for $\mathrm{H}, \mathrm{H}_{2}$, and $\mathrm{CO}$.

\begin{tabular}{clcc}
\hline \hline No. & Reaction ${ }^{(a)}$ & $a$ & $b$ \\
\hline 1. & $\mathrm{H}_{2}+\mathrm{CR} \rightarrow \mathrm{H}_{2}^{+}+\mathrm{e}^{-}$ & $\ldots{ }^{(b)}$ & $\ldots$ \\
2. & $\mathrm{H}_{2}^{+}+\mathrm{H}_{2} \rightarrow \mathrm{H}_{3}^{+}+\mathrm{H}$ & $2 \times 10^{-9}$ & 0 \\
3. & $\mathrm{H}_{3}^{+}+\mathrm{N}_{2} \rightarrow \mathrm{N}_{2} \mathrm{H}^{+}+\mathrm{H}_{2}$ & $1.70 \times 10^{-9}$ & 0 \\
4. & $\mathrm{H}_{3}^{+}+\mathrm{CO} \rightarrow \mathrm{HCO}^{+}+\mathrm{H}_{2}$ & $1.61 \times 10^{-9}$ & 0 \\
5. & $\mathrm{N}_{2} \mathrm{H}^{+}+\mathrm{CO} \rightarrow \mathrm{HCO}^{+}+\mathrm{N}_{2}$ & $8.8 \times 10^{-10}$ & 0 \\
6. & $\mathrm{HCO}^{+}+\mathrm{e}^{-} \rightarrow \mathrm{H}+\mathrm{CO}$ & $2.8 \times 10^{-7}$ & -0.69 \\
7. & $\mathrm{N}_{2} \mathrm{H}^{+}+\mathrm{e}^{-} \rightarrow \mathrm{NH}+\mathrm{N}$ & $1.3 \times 10^{-8}$ & -0.84 \\
8. & $\mathrm{N}_{2} \mathrm{H}^{+}+\mathrm{e}^{-} \rightarrow \mathrm{N}_{2}+\mathrm{H}$ & $2.47 \times 10^{-7}$ & -0.84 \\
9. & $\mathrm{H}_{3}^{+}+\mathrm{e}^{-} \rightarrow \mathrm{H}_{2}+\mathrm{H}$ & $2.34 \times 10^{-8}$ & -0.52 \\
10. & $\mathrm{H}_{3}++\mathrm{e}^{-} \rightarrow \mathrm{H}+\mathrm{H}+\mathrm{H}$ & $4.36 \times 10^{-8}$ & -0.52 \\
\hline
\end{tabular}

Notes. Reactions with a cosmic ray (CR) is also included. Reaction rate coefficients are obtained from the Kinetic Database for Astrochemistry (KiDA, Wakelam et al. 2012). ${ }^{(a)}$ The reaction rate coefficient is given by $k=a \times(T / 300 \mathrm{~K})^{b} \mathrm{~cm}^{3} \mathrm{~s}^{-1}$. These reaction rates are valid up to $300 \mathrm{~K}$. ${ }^{(b)}$ See text for the CR ionization rate.

deeper and spatially resolved $\mathrm{HCN}$ and water observations are needed to fully reveal the physical processes that can cause these variations.

\subsection{Accretion processes in a young disk}

During the formation of a star and disk, mass flows from the large-scale envelope to the disk and the young protostar. Previously, disk accretion rates have been measured through the bolometric luminosity and/or molecular emission at large radii ( $>500 \mathrm{au}$ ). On the other hand, a detailed 2D model of a bursting Class I protostar can capture the current disk structure and infer the more accurate accretion rate during the burst (e.g., Baek et al. 2020; Lee et al. 2020). From the bolometric luminosity of TMC1A $\left(L_{\text {bol }}=2.7 L_{\odot}\right.$, Kristensen et al. 2012), the accretion rate is inferred to be $\sim 3 \times 10^{-7} M_{\odot} \mathrm{yr}^{-1}$. Similar values are derived from molecular line observations (e.g., Aso et al. 2015; Mottram et al. 2017). These inferred accretion rates usually refer to the mass flow from envelope-to-disk or from disk-to-star (i.e. stellar). With our spatially resolved molecular line observations and associated analysis, it is possible to re-examine the accretion rate and investigate the dominant accretion mechanism in the TMC1A disk.

An accretion disk mediates the mass transfer between the large-scale envelope and the young protostar. It does so by shifting angular momentum from the accreting the mass, which, e.g., can result in a viscously growing disc. One of the major uncertainties and sources of continued debate in understanding the evolution of disks is the physical driver of accretion. A parameterized and constant viscosity ( $\alpha$, Shakura \& Sunyaev 1973 ) is typically adopted to describe the transport of angular momentum through the disk (e.g., Hueso \& Guillot 2005; Visser et al. 2009), but this alone does not reveal the physical driver. In rotating magnetized disks, the magnetorotational instability (MRI, Balbus \& Hawley 1991; Balbus 2003) can drive accretion (and turbulence) if the disk is sufficiently ionized (e.g., Balbus \& Hawley 2000). These proposed theories rely on the kinematical structure of the disk being nearly Keplerian. In order to constrain whether MRI is active in the TMC1A disk, which is indeed a Keplerian disk, we need to estimate the ionization fraction, and $\mathrm{HCO}^{+}$can be used to do this.
The abundance of $\mathrm{HCO}^{+}$is chemically linked to the electron abundance, $X_{\mathrm{e}}$, which can be used to determine the ionization fraction. A simple chemical model that links $\mathrm{CO}$ to $\mathrm{HCO}^{+}$and $\mathrm{N}_{2} \mathrm{H}^{+}$can be found in Aikawa et al. (2015, but see also Jørgensen et al. 2004). Table 4 lists the reaction network from Aikawa et al. (2015) that we employ here. We adopt a fixed $\mathrm{CO}$ abundance of $10^{-4}$ and a $\mathrm{N}_{2}$ abundance of $3 \times 10^{-6}$ relative to $\mathrm{H}_{2}$. The high relative abundance of $\mathrm{CO}$ leads to it dominating the chemistry, and the adopted value of $\mathrm{N}_{2}$ abundance does not affect our results. We compute the electron number density from the cosmic ray (CR) ionization rate $\zeta$ using $n_{\mathrm{e}^{-}}=2 \sqrt{\zeta /\left(2 k_{6} n_{\mathrm{H}}\right)} n_{H_{2}}$ (Aikawa et al. 2015) where $k_{6}$ is the rate coefficient of the sixth reaction in Table 4. A range of $\mathrm{H}_{2}$ densities, temperature, and $\zeta$ values are explored to investigate the effect on the resulting $\mathrm{HCO}^{+}$abundance. With $\mathrm{CO}, \mathrm{H}_{2}, \mathrm{~N}_{2}$, and $\mathrm{e}^{-}$abundances known, we solve for the equilibrium abundance of $\mathrm{HCO}^{+}$.

Using these approximations, the inferred $\mathrm{HCO}^{+}$abundance can be reproduced with $\zeta \sim 10^{-17} \mathrm{~s}^{-1}, \mathrm{H}_{2}$ densities of $10^{6} \mathrm{~cm}^{-3}$, and a gas temperature of $20 \mathrm{~K}$. We find that the gas density $n_{\mathrm{H}_{2}}$ is the dominant factor in the calculation, while the gas temperature does not strongly affect the results; the variance in $\mathrm{HCO}^{+}$abundance is less than a factor of 2 for temperatures between 20 and $100 \mathrm{~K}$. From varying the $\mathrm{H}_{2}$ density, we find that $\mathrm{HCO}^{+}$emission seems to be confined to regions with $n_{\mathrm{H}_{2}}<10^{8} \mathrm{~cm}^{-3}$ (see Mathews et al. 2013).

An MRI active region is defined by its magnetic Reynolds number

$R_{\mathrm{e}}=\frac{c_{\mathrm{s}} h}{D} \approx 1\left(\frac{X_{\mathrm{e}}}{10^{-13}}\right) T_{100 \mathrm{~K}}^{1 / 2} R_{\mathrm{au}}^{3 / 2}$,

where $c_{\mathrm{s}}$ is the sound speed, $h$ is the disk scale height, $D$ is the magnetic diffusivity, $T_{100 \mathrm{~K}}$ is the gas temperature normalized to $100 \mathrm{~K}$, and $R_{\text {au }}$ is the radial distance from the star normalized to 1 au (Perez-Becker \& Chiang 2011). A secondary condition for a MRI unstable disk is a high ion-neutral collision rate, i.e., that the turbulence is efficiently transferred to the bulk neutral disk. The ion-neutral collision rate can be expressed using

$\mathrm{Am} \approx 1\left(\frac{X_{i}}{10^{-8}}\right) n_{10} R_{\mathrm{au}}^{3 / 2}$

where $X_{i}$ is the abundance of a singly ionized species and $n_{10}$ is the gas number density normalized to $10^{10} \mathrm{~cm}^{-3}$ (Perez-Becker \& Chiang 2011). Here, we assume that $\mathrm{HCO}^{+}$is the dominant ion. Given the estimated abundance of the $\mathrm{HCO}^{+}$emitting layer, and the inferred electron abundance $X_{\mathrm{e}}$ (using $n_{\mathrm{H}_{2}}=10^{6} \mathrm{~cm}^{-3}$ and $\zeta=10^{-17} \mathrm{~s}^{-1}$; see above), we estimate that $R_{\mathrm{e}}>10^{6}$ and Am $<0.1$. Depending on the disk structure and the magnetic field orientation, the region of the disk needs to be above the critical $R_{\mathrm{e}}=10^{2}-10^{4}$ (Fleming et al. 2000; Flock et al. 2012) for MRI to be active. Magneto-hydrodynamic disk shearing box simulations by Bai \& Stone (2011) meanwhile suggest that MRI can be active in disks at any given Am if the disk is sufficiently weakly magnetized. Therefore, unless the TMC1A disk is weakly magnetized, which is in contrast to the magneticallypowered disk wind observed in TMC1A (Bjerkeli et al. 2016), MRI is likely not active in the observed molecular layer.

The current disk accretion rate can also be inferred through the location of the water snow surface. We use the brightness temperature profile in Fig. 10 to estimate that the water snow surface in TMC1A is located at $\simeq 10$ au (i.e. where $T<$ $160 \mathrm{~K}$ ), which is consistent with the non-detection of millimeter $\mathrm{H}_{2}^{18} \mathrm{O}$ emission from this disk (Harsono et al. 2020). The 
relation between the midplane water snowline and the disk accretion rate in embedded disks was explored in Harsono et al. (2015). From those results, and the non-detection of the water line, we infer that the stellar accretion rate is $\lesssim 10^{-5} M_{\odot} \mathrm{yr}^{-1}$ in TMC1A.

The observed brightness temperature profile (Sect. 4.2) likely traces the irradiated disk surface $\left(T_{\text {eff }} \sim L_{\star}^{1 / 4} R^{-1 / 2}\right)$. From the inferred water snowline location and the current bolometric luminosity, we estimate that the current stellar accretion rate is close to $10^{-6} M_{\odot} \mathrm{yr}^{-1}$. Note that the current TMC1A disk accretion rate $\left(\sim 10^{-6} M_{\odot} \mathrm{yr}^{-1}\right)$ is higher than the values obtained from its bolometric luminosity and previous large-scale molecular emission. A more detailed 2D physical structure (see e.g., Cleeves et al. 2013, 2017) of the disk+envelope is, however, required to more accurately assess these values.

Other sources of angular momentum transport which could drive the accretion in the TMC1A disk could be magneticallypowered winds (e.g. Bai 2013; Ramsey \& Clarke 2019), and gravitational (GI) or hydrodynamical instabilities (Lyra \& Umurhan 2019). Due to the absence of observable dust substructures in TMC1A, Harsono et al. (2018) suggest that $M_{\text {disk }} / M_{\star} \lesssim 0.1$, yielding $\alpha_{\mathrm{GI}} \lesssim 0.06$ (i.e. a long cooling timescale $t_{\text {cool }} \sim 10 \Omega^{-1}$ ).

\section{Summary and conclusions}

This paper presents spatially resolved observations of dust and gas with ALMA toward the young disk around TMC1A. The high-spatial resolution provided by $16 \mathrm{~km}$ baselines has proven crucial in isolating the emission of the young disk from its surrounding envelope. Studies such as this are critical to tracing the evolution of various molecules during the early stages of planet formation. The results and conclusions of this paper are as follows.

- The dust disk is detected at 203, 220, 230, 240, and $260 \mathrm{GHz}$.

Dust continuum visibilities are analyzed with Gaussian intensity profiles at each frequency to constrain the orientation of the disk around TMC1A to $i=50^{\circ} \pm 3^{\circ}$ and $\mathrm{PA}=76^{\circ} \pm 4^{\circ}$.

- We present high-spatial observations of DCN 3-2, HCN $3-2, \mathrm{HCO}^{+} 3-2$, and $\mathrm{SO}_{5}-45$, as well as a hint of spectrally unresolved $\mathrm{N}_{2} \mathrm{D}^{+}$emission. The $\mathrm{N}_{2} \mathrm{D}^{+}$emission cannot be imaged because it is mostly filtered out.

- High-spatial-resolution CO observations are essential to distinguish the molecular emission associated with the disk wind, the envelope, and the Keplerian disk. By comparing the morphology of the $\mathrm{HCO}^{+}$emission to $\mathrm{CO}$, we determine that $\mathrm{HCO}^{+}$traces the upper layers of the disk and parts of the infalling envelope.

- Two HCN emission peaks are located to the west on the redshifted side of the disk and to the north of the blue-shifted side of the disk. By comparing the $\mathrm{HCN}$ to ${ }^{12} \mathrm{CO}$ and $\mathrm{C}^{18} \mathrm{O}$, the narrow line profile suggests that the emission to the north of the disk traces the protostellar envelope near the outflow cavity wall. Meanwhile, the red-shifted HCN emission to the west is co-spatial with the $\mathrm{HCO}^{+}$emission and emanates from the surface of the embedded Keplerian disk.

- The zeroth moment map of SO shows a partial ring-like structure that has been seen towards other protostellar systems. Owing to the orientation of the TMC1A system, we are able to differentiate between the plane of SO emission and the Keplerian disk as traced by $\mathrm{C}^{18} \mathrm{O}$. The combination of the SO line profile and its low brightness temperature indicates that the emission originates from the dense and warm inner envelope close to the outflow cavity wall.
- The molecular emission is analyzed considering thermalized, optically thin emission as well as non-LTE models using the RADEX radiative transfer code. We find that the optically thin limit provides a reasonable estimate of the emitting column densities of the detected molecular lines. With the derived column densities, we infer the abundance structure of the disk relative to $\mathrm{CO}$. The disk-averaged abundances are then $X_{\mathrm{HCO}^{+}}=10^{-10}-10^{-9}, X_{\mathrm{HCN}}=10^{-11}-10^{-9}$ and $X_{\mathrm{SO}}=10^{-9}-10^{-7}$. With an upper limit to the DCN emission, we estimate a DCN/HCN ratio of $<0.26$ for the TMC1A disk.

- The comparison of molecular abundances of the disk to its surrounding envelope shows that the observed molecular layer of the disk is composed of unaltered infalling gas. This indicates a smooth transition between the envelope and the young disk. Furthermore, the similarity of the relative abundances of $\mathrm{HCO}^{+}, \mathrm{HCN}$, and $\mathrm{SO}$ between the young TMC1A disk and Class II disks suggest that some parts of the molecular layer in disks are set during the disk formation phase.

- The derived $\mathrm{HCN}, \mathrm{DCN}$, and $\mathrm{H}_{2} \mathrm{O}$ molecular abundances of the TMC1A disk show larger discrepancies relative to Class II disks and Solar System objects (comet 67P, Hale Bopp, and Titan). While the $\mathrm{HCN} / \mathrm{H}_{2} \mathrm{O}$ ratio of the TMC1A disk is consistent with observed inner disks and comets, the ratio is different from values typically found in protostellar envelopes. Similarly, the individual HCN abundance of the TMC1A disk is different from Solar System comets despite that it is within the range of other Class II disks. From these comparisons, it would seem that the ratio of $\mathrm{HCN} / \mathrm{H}_{2} \mathrm{O}$ is established during the disk formation process. We propose that deeper observations of $\mathrm{HCN}$ isotopologs and $\mathrm{H}_{2} \mathrm{O}$ are crucial to understand the early physical and chemical evolution of planet-forming disks.

- Explaining the accretion process in disks is one of the fundamental problems in astrophysics. With the derived $\mathrm{HCO}^{+}$ abundance, we find that the observed molecular layer of the TMC1A disk is not sufficiently ionized to be MRI unstable. The ionization rate is obtained using a reduced chemical network to reproduce the observed $\mathrm{HCO}^{+}$abundance. We estimate an accretion rate of the TMC1A disk of $\sim 10^{-6} M_{\odot} \mathrm{yr}^{-1}$. Other physical processes such as disk winds, gravitational instability, or hydrodynamical instabilities are thus needed to drive accretion in TMC1A.

These results are one of the first that directly compare the relative molecular abundances (six species) in a young disk with its own envelope, Class II disks, and Solar System objects. The aim of this comparison is to provide molecular tracers that can probe the physics of disk formation and accretion. In addition, $\mathrm{HCO}^{+}$ and $\mathrm{H}_{2} \mathrm{O}$ observations are crucial in revealing the accretion process (envelope-to-disk, disk-to-star) during the embedded stage of star formation. These observations support the idea that the composition of the molecular layer of planet-forming disks is determined during the disk formation phase. Future deep observations that require ALMA LBC ( $>16 \mathrm{~km}$ baselines) per target $\left(L_{\text {bol }} \sim 3 L_{\odot}\right)$ within 200 pc will be needed to further unravel the chemical structure of Keplerian disks around young stellar objects.

Acknowledgements. This paper makes use of the following ALMA data: ADS/JAO.ALMA\#2015.1.01549.S, ADS/JAO.ALMA\#2016.1.00711.S, and ADS/JAO.ALMA\#2017.1.00212.S. ALMA is a partnership of ESO (representing its member states), NSF (USA) and NINS (Japan), together with NRC (Canada) and NSC and ASIAA (Taiwan) and KASI (Republic of Korea), in cooperation with the Republic of Chile. The Joint ALMA Observatory is operated by 
ESO, AUI/NRAO and NAOJ. This work uses observations carried out under project number V064 and X065 with the IRAM NOEMA Interferometer/PdBI. IRAM is supported by INSU/CNRS (France), MPG (Germany) and IGN (Spain). This work is based on observations collected at the European Southern Observatory Very Large Telescope under program ID 179.C-0151. We thank Klaus Pontoppidan and Greg Herczeg for providing the calibrated data. We thank the anonymous referee for carefully reading the manuscript and their constructive feedback that improved this paper. Astrochemistry in Leiden is supported by the European Union A-ERC grant 291141 CHEMPLAN, by the Netherlands Research School for Astronomy (NOVA) and by a Royal Netherlands Academy of Arts and Sciences (KNAW) professor prize. D.H acknowledges support from the EACOA Fellowship from the East Asian Core Observatories Association. P.B. acknowledges the support of the Swedish Research Council (VR) through contracts 2013-00472 and 2017-04924. J.P.R acknowledges support from Virginia Initiative on Cosmic Origins (VICO), and the National Science Foundation (NSF) under grant nos. AST-1910106 and AST-1910675. J.K.J. acknowledges support by the European Research Council (ERC) under the European Union's Horizon 2020 research and innovation programme through Cosolidator Grant "S4F" (grant agreement No 646908). This research made use of Astropy community-developed Python package for Astronomy (Astropy Collaboration 2018), numpy, matplotlib (Hunter 2007), and python package CASACORE to handle CASA products (images and measurement sets).

\section{References}

Aikawa, Y., Umebayashi, T., Nakano, T., \& Miyama, S. M. 1999, ApJ, 519, 705

Aikawa, Y., Momose, M., Thi, W.-F., et al. 2003, PASJ, 55, 11

Aikawa, Y., Furuya, K., Nomura, H., \& Qi, C. 2015, ApJ, 807, 120

ALMA Partnership, Brogan, C. L., Pérez, L. M., et al. 2015, ApJ, 808, L3

Armitage, P. J. 2011, ARA\&A, 49, 195

Artur de la Villarmois, E., Kristensen, L. E., Jørgensen, J. K., et al. 2018, A\&A, 614, A26

Artur de la Villarmois, E., Jørgensen, J. K., Kristensen, L. E., et al. 2019a, A\&A, 626, A71

Artur de la Villarmois, E., Kristensen, L. E., \& Jørgensen, J. K. 2019b, A\&A, 627, A37

Aso, Y., Ohashi, N., Saigo, K., et al. 2015, ApJ, 812, 27

Asplund, M., Grevesse, N., Sauval, A. J., \& Scott, P. 2009, ARA\&A, 47, 481

Astropy Collaboration (Price-Whelan, A. M., et al.) 2018, AJ, 156, 123

Baek, G., MacFarlane, B. A., Lee, J.-E., et al. 2020, ApJ, 895, 27

Bai, X.-N. 2013, ApJ, 772, 96

Bai, X.-N., \& Stone, J. M. 2011, ApJ, 736, 144

Balbus, S. A. 2003, ARA\&A, 41, 555

Balbus, S. A., \& Hawley, J. F. 1991, ApJ, 376, 214

Balbus, S. A., \& Hawley, J. F. 2000, Space Sci. Rev., 92, 39

Bast, J. E., Brown, J. M., Herczeg, G. J., van Dishoeck, E. F., \& Pontoppidan, K. M. 2011, A\&A, 527, A119

Bergin, E. A., \& Langer, W. D. 1997, ApJ, 486, 316

Bergin, E. A., Hogerheijde, M. R., Brinch, C., et al. 2010, A\&A, 521, L33

Bisschop, S. E., Jørgensen, J. K., Bourke, T. L., Bottinelli, S., \& van Dishoeck, E. F. 2008, A\&A, 488, 959

Bjerkeli, P., van der Wiel, M. H. D., Harsono, D., Ramsey, J. P., \& Jørgensen, J. K. 2016, Nature, 540, 406

Blake, G. A., Sandell, G., van Dishoeck, E. F., et al. 1995, ApJ, 441, 689

Bockelée-Morvan, D., Lis, D. C., Wink, J. E., et al. 2000, A\&A, 353, 1101

Bollard, J., Connelly, J. N., Whitehouse, M. J., et al. 2017, Sci. Adv., 3, e1700407

Booth, A. S., Walsh, C., \& Ilee, J. D. 2019, A\&A, 629, A75

Bosman, A. D., Walsh, C., \& van Dishoeck, E. F. 2018, A\&A, 618, A182

Bosman, A. D., Cridland, A. J., \& Miguel, Y. 2019, A\&A, 632, L11

Botschwina, P., Horn, M., Flügge, J., \& Seeger, S. 1993, J. Chem. Soc. Faraday Trans., 89, 2219

Briggs, D. S. 1995, BAAS, 27, 1444

Bruderer, S., van Dishoeck, E. F., Doty, S. D., \& Herczeg, G. J. 2012, A\&A, 541 , A91

Bruderer, S., Harsono, D., \& van Dishoeck, E. F. 2015, A\&A, 575, A94

Calvet, N., Hartmann, L., \& Hewett, R. 1992, ApJ, 386, 229

Carney, M. T., Fedele, D., Hogerheijde, M. R., et al. 2018, A\&A, 614, A106

Carney, M. T., Hogerheijde, M. R., Guzmán, V. V., et al. 2019, A\&A, 623, A124

Carr, J. S., \& Najita, J. R. 2008, Science, 319, 1504

Caselli, P., Keto, E., Bergin, E. A., et al. 2012, ApJ, 759, L37

Ceccarelli, C., Dominik, C., Caux, E., Lefloch, B., \& Caselli, P. 2005, ApJ, 631, L81

Ceccarelli, C., Hollenbach, D. J., \& Tielens, A. G. G. M. 1996, ApJ, 471, 400

Chapillon, E., Guilloteau, S., Dutrey, A., Piétu, V., \& Guélin, M. 2012, A\&A, 537, A60

Chiang, E. I., \& Goldreich, P. 1997, ApJ, 490, 368
Cleeves, L. I., Adams, F. C., \& Bergin, E. A. 2013, ApJ, 772, 5

Cleeves, L. I., Bergin, E. A., Öberg, K. I., et al. 2017, ApJ, 843, L3

Cleeves, L. I., Öberg, K. I., Wilner, D. J., et al. 2018, ApJ, 865, 155

Connelly, J. N., Bizzarro, M., Krot, A. N., et al. 2012, Science, 338, 651

Crapsi, A., Caselli, P., Walmsley, C. M., et al. 2005, ApJ, 619, 379

Cuzzi, J. N., \& Alexander, C. M. O. 2006, Nature, 441, 483

Dodson-Robinson, S. E., Evans, Neal J., I., Ramos, A., Yu, M., \& Willacy, K. 2018, ApJ, 868, L37

Doty, S. D., van Dishoeck, E. F., van der Tak, F. F. S., \& Boonman, A. M. S. 2002, A\&A, 389, 446

Drozdovskaya, M. N., van Dishoeck, E. F., Jørgensen, J. K., et al. 2018, MNRAS, 476, 4949

Drozdovskaya, M. N., van Dishoeck, E. F., Rubin, M., Jørgensen, J. K., \& Altwegg, K. 2019, MNRAS, 490, 50

Du, F., Bergin, E. A., Hogerheijde, M., et al. 2017, ApJ, 842, 98

Dutrey, A., Guilloteau, S., \& Guelin, M. 1997, A\&A, 317, L55

Endres, C. P., Schlemmer, S., Schilke, P., Stutzki, J., \& Müller, H. S. 2016, J. Mol. Spectr., 327, 95

Fedele, D., van Dishoeck, E. F., Kama, M., Bruderer, S., \& Hogerheijde, M. R. 2016, A\&A, 591, A95

Fleming, T. P., Stone, J. M., \& Hawley, J. F. 2000, ApJ, 530, 464

Flock, M., Henning, T., \& Klahr, H. 2012, ApJ, 761, 95

Foreman-Mackey, D., Hogg, D. W., Lang, D., \& Goodman, J. 2013, PASP, 125, 306

Garrod, R. T. 2019, ApJ, 884, 69

Goldsmith, P. F., \& Langer, W. D. 1999, ApJ, 517, 209

Graninger, D. M., Wilkins, O. H., \& Öberg, K. I. 2016, ApJ, 819, 140

Green, S., \& Thaddeus, P. 1974, ApJ, 191, 653

Guzmán, V. V., Öberg, K. I., Loomis, R., \& Qi, C. 2015, ApJ, 814, 53

Harsono, D., Jørgensen, J. K., van Dishoeck, E. F., et al. 2014, A\&A, 562, A77

Harsono, D., Bruderer, S., \& van Dishoeck, E. F. 2015, A\&A, 582, A41

Harsono, D., Bjerkeli, P., van der Wiel, M. H. D., et al. 2018, Nat. Astron., 2, 646

Harsono, D., Persson, M. V., Ramos, A., et al. 2020, A\&A, 636, A26

Haugbølle, T., Weber, P., Wielandt, D. P., et al. 2019, AJ, 158, 55

Herczeg, G. J., Brown, J. M., van Dishoeck, E. F., \& Pontoppidan, K. M. 2011, A\&A, 533, A112

Hincelin, U., Wakelam, V., Commerc con, B., Hersant, F., \& Guilloteau, S. 2013, ApJ, 775, 44

Huang, J., Öberg, K. I., Qi, C., et al. 2017, ApJ, 835, 231

Huang, J., Andrews, S. M., Pérez, L. M., et al. 2018, ApJ, 869, L43

Hueso, R., \& Guillot, T. 2005, A\&A, 442, 703

Hunter, J. D. 2007, Comput. Sci. Eng., 9, 90

Jacquet, E., Pignatale, F. C., Chaussidon, M., \& Charnoz, S. 2019, ApJ, 884, 32

Jankowski, P., \& Szalewicz, K. 2005, J. Chem. Phys., 123, 104301

Jørgensen, J. K., Schöier, F. L., \& van Dishoeck, E. F. 2004, A\&A, 416, 603

Jørgensen, J. K., Bourke, T. L., Myers, P. C., et al. 2005, ApJ, 632, 973

Jørgensen, J. K., Bourke, T. L., Myers, P. C., et al. 2007, ApJ, 659, 479

Jørgensen, J. K., van Dishoeck, E. F., Visser, R., et al. 2009, A\&A, 507, 861

Jørgensen, J. K., Visser, R., Sakai, N., et al. 2013, ApJ, 779, L22

Kalyaan, A., \& Desch, S. J. 2019, ApJ, 875, 43

Kama, M., Bruderer, S., van Dishoeck, E. F., et al. 2016, A\&A, 592, A83

Krijt, S., Ciesla, F. J., \& Bergin, E. A. 2016, ApJ, 833, 285

Kristensen, L. E., van Dishoeck, E. F., Bergin, E. A., et al. 2012, A\&A, 542, A8

Lee, Y.-H., Johnstone, D., Lee, J.-E., et al. 2020, ApJ, 903, 5

Lique, F., Spielfiedel, A., Dhont, G., \& Feautrier, N. 2006, A\&A, 458, 331

Lodato, G. 2008, New A Rev., 52, 21

Long, F., Pinilla, P., Herczeg, G. J., et al. 2018, ApJ, 869, 17

Loomis, R. A., Cleeves, L. I., Öberg, K. I., Guzman, V. V., \& Andrews, S. M. 2015, ApJ, 809, L25

Lyra, W., \& Umurhan, O. M. 2019, PASP, 131, 072001

Manara, C. F., Testi, L., Herczeg, G. J., et al. 2017, A\&A, 604, A127

Manara, C. F., Mordasini, C., Testi, L., et al. 2019, A\&A, 631, L2

Mangum, J. G., \& Shirley, Y. L. 2015, PASP, 127, 266

Mathews, G. S., Klaassen, P. D., Juhász, A., et al. 2013, A\&A, 557, A132

Maud, L. T., Tilanus, R. P. J., van Kempen, T. A., et al. 2017, A\&A, 605, A121

Maury, A. J., Belloche, A., André, P., et al. 2014, A\&A, 563, L2

McClure, M. K. 2019, A\&A, 632, A32

McClure, M. K., Bergin, E. A., Cleeves, L. I., et al. 2016, ApJ, 831, 167

McMullin, J. P., Waters, B., Schiebel, D., Young, W., \& Golap, K. 2007, ASP Conf. Ser., 376, 127

Meier, R., Owen, T. C., Jewitt, D. C., et al. 1998, Science, 279, 1707

Meijerink, R., Pontoppidan, K. M., Blake, G. A., Poelman, D. R., \& Dullemond, C. P. 2009, ApJ, 704, 1471

Miotello, A., van Dishoeck, E. F., Williams, J. P., et al. 2017, A\&A, 599, A113

Molter, E. M., Nixon, C. A., Cordiner, M. A., et al. 2016, AJ, 152, 42

Mottram, J. C., van Dishoeck, E. F., Kristensen, L. E., et al. 2017, A\&A, 600, A99 
Müller, H. S. P., Schlöder, F., Stutzki, J., \& Winnewisser, G. 2005, J. Mol. Struc., 742,215

Mullins, A. M., Loughnane, R. M., Redman, M. P., et al. 2016, MNRAS, 459, 2882

Najita, J. R., Carr, J. S., Pontoppidan, K. M., et al. 2013, ApJ, 766, 134

Najita, J. R., Carr, J. S., Salyk, C., et al. 2018, ApJ, 862, 122

Öberg, K. I., \& Wordsworth, R. 2019, AJ, 158, 194

Öberg, K. I., Guzmán, V. V., Merchantz, C. J., et al. 2017, ApJ, 839, 43

Owen, T., Mahaffy, P., Niemann, H. B., et al. 1999, Nature, 402, 269

Perez-Becker, D., \& Chiang, E. 2011, ApJ, 735, 8

Pérez, L. M., Carpenter, J. M., Andrews, S. M., et al. 2016, Science, 353, 1519

Persson, M. V., Jørgensen, J. K., van Dishoeck, E. F., \& Harsono, D. 2014, A\&A, 563, A74

Pickett, H. M., Poynter, R. L., Cohen, E. A., et al. 1998, J. Quant. Spectr. Rad. Transf., 60, 883

Piétu, V., Dutrey, A., \& Guilloteau, S. 2007, A\&A, 467, 163

Pontoppidan, K. M., Blake, G. A., \& Smette, A. 2011, ApJ, 733, 84

Pontoppidan, K. M., Salyk, C., Bergin, E. A., et al. 2014, in Protostars and Planets

VI, eds. H. Beuther, C. Dullemond, \& T. Henning (Tucson: University of Arizona Press ), 363

Ramsey, J. P., \& Clarke, D. A. 2019, MNRAS, 484, 2364

Roberts, H., Fuller, G. A., Millar, T. J., Hatchell, J., \& Buckle, J. V. 2002, A\&A, 381, 1026

Robitaille, T. P., Whitney, B. A., Indebetouw, R., Wood, K., \& Denzmore, P. 2006, ApJS, 167, 256

Rubin, M., Altwegg, K., Balsiger, H., et al. 2019, MNRAS, 489, 594

Sakai, N., Oya, Y., Sakai, T., et al. 2014a, ApJ, 791, L38

Sakai, N., Sakai, T., Hirota, T., et al. 2014b, Nature, 507, 78

Salinas, V. N., Hogerheijde, M. R., Bergin, E. A., et al. 2016, A\&A, 591, A122

Salyk, C., Pontoppidan, K. M., Blake, G. A., Najita, J. R., \& Carr, J. S. 2011 ApJ, 731, 130

Schmalzl, M., Visser, R., Walsh, C., et al. 2014, A\&A, 572, A81

Schöier, F. L., Jørgensen, J. K., van Dishoeck, E. F., \& Blake, G. A. 2002, A\&A, 390, 1001
Schöier, F. L., van der Tak, F. F. S., van Dishoeck, E. F., \& Black, J. H. 2005, A\&A, 432, 369

Shakura, N. I., \& Sunyaev, R. A. 1973, A\&A, 24, 337

Tabone, B., Cabrit, S., Bianchi, E., et al. 2017, A\&A, 607, L6

Tafalla, M., Santiago-García, J., Myers, P. C., et al. 2006, A\&A, 455, 577

Taquet, V., López-Sepulcre, A., Ceccarelli, C., et al. 2015, ApJ, 804, 81

Tazzari, M., Testi, L., Natta, A., et al. 2017, A\&A, 606, A88

Tazzari, M., Beaujean, F., \& Testi, L. 2018, MNRAS, 476, 4527

Teague, R., Semenov, D., Guilloteau, S., et al. 2015, A\&A, 574, A137

Tobin, J. J., Hartmann, L., Chiang, H.-F., et al. 2013, ApJ, 771, 48

van Dishoeck, E. F., \& Blake, G. A. 1998, ARA\&A, 36, 317

van der Tak, F. F. S., Black, J. H., Schöier, F. L., Jansen, D. J., \& van Dishoeck, E. F. 2007, A\&A, 468, 627

van der Marel, N., van Dishoeck, E. F., Bruderer, S., \& van Kempen, T. A. 2014, A\&A, 563, A113

van 't Hoff, M. L. R., Persson, M. V., Harsono, D., et al. 2018a, A\&A, 613, A29

van 't Hoff, M. L. R., Tobin, J. J., Harsono, D., \& van Dishoeck, E. F. 2018b, A\&A, 615, A83

van 't Hoff, M. L. R., Harsono, D., Tobin, J. J., et al. 2020, ApJ, 901, 166

van Zadelhoff, G. J., van Dishoeck, E. F., Thi, W. F., \& Blake, G. A. 2001, A\&A, 377,566

Visser, R., van Dishoeck, E. F., Doty, S. D., \& Dullemond, C. P. 2009, A\&A, 495,881

Wakelam, V., Herbst, E., Loison, J.-C., et al. 2012, ApJS, 199, 21

Willacy, K. 2007, ApJ, 660, 441

Williams, J. P., \& Cieza, L. A. 2011, ARA\&A, 49, 67

Wilson, T. L., \& Rood, R. 1994, ARA\&A, 32, 191

Yang, B., Stancil, P. C., Balakrishnan, N., \& Forrey, R. C. 2010, ApJ, 718, 1062

Yen, H.-W., Koch, P. M., Takakuwa, S., et al. 2017, ApJ, 834, 178

Yoneda, H., Tsukamoto, Y., Furuya, K., \& Aikawa, Y. 2016, ApJ, 833, 105

Yu, M., Evans, Neal J., I., Dodson-Robinson, S. E., Willacy, K., \& Turner, N. J. 2017, ApJ, 841, 39

Zhang, K., Schwarz, K. R., \& Bergin, E. A. 2020, ApJ, 891, L17 


\section{Appendix A: Undetected molecular lines}

Table A.1. Undetected molecular lines in our spectral windows.

\begin{tabular}{lccccc}
\hline \hline Molecular line transition & $\begin{array}{c}\text { Frequency } \\
(\mathrm{GHz})\end{array}$ & $\begin{array}{c}E_{\mathrm{up}} \\
(\mathrm{K})\end{array}$ & $\begin{array}{c}\log _{10} A_{\mathrm{ul}} \\
\left(\mathrm{s}^{-1}\right)\end{array}$ & $\begin{array}{c}\text { Beam }^{(a)} \\
\left({ }^{\prime \prime} \times^{\prime \prime},{ }^{\circ}\right)\end{array}$ & $\begin{array}{c}\text { Noise } \\
\left(\mathrm{mJy} \mathrm{bm}^{-1}\right)\end{array}$ \\
\hline $\mathrm{SiO} J=5-4$ & 217.10450 & 31.26 & -3.28 & $0.13 \times 0.10,32$ & 1.6 \\
${ }^{13} \mathrm{CN} N_{J}=2_{3 / 2}-1_{1 / 2}, F_{1}, F=1,1-0,1$ & 217.27768 & 10.88 & -4.24 & $0.08 \times 0.07,78$ & $\sim 5$ \\
${ }^{13} \mathrm{CS} J=5-4$ & 231.22069 & 33.29 & -3.60 & $0.12 \times 0.10,32$ & 1 \\
$\mathrm{SO} N_{J}=3_{4}-4$ & 267.19774 & 19.93 & -6.15 & $0.13 \times 0.10,3$ & 1.3 \\
\hline
\end{tabular}

Notes. Synthesized beams and noise levels per velocity channel $\left(0.3 \mathrm{~km} \mathrm{~s}^{-1}\right)$ are shown. ${ }^{(a)}$ Elliptical synthesized beam parametrized by: FWHM long axis $\times$ FWHM short axis, position angle of the long axis.

There are additional molecular lines present in our ALMA spectral set ups that are not detected in our data. Table A.1 lists these lines. The ${ }^{13} \mathrm{CN}$ line is weakly apparent in the visibilities and could be identified in a spectrum taken over a large area ( $>10$ beams). However, it cannot be imaged properly even including some tapering. The noise level per channel is higher than the other lines because low-level ${ }^{13} \mathrm{CN}$ permeates the spectral cube. This implies that most of the emission is filtered out in our long baseline data. SO $N_{J}=3_{4}-4_{3}$ is likely not detected because of its low Einstein A coefficient; for the physical conditions that produce the detected SO $5_{6}-4_{5}$ line, the strength of the $3_{4}-4_{3}$ line would be a factor of 100 weaker.

\section{Appendix B: Molecular lines: vibrational transitions}

Our spectral cubes also contain the $\mathrm{HCN}$ bending mode $\left(v_{2}=1\right)$ rotational transitions at 265.8527 and $267.1993 \mathrm{GHz}$, as shown in Fig. B.1. These lines are not detected toward TMC1A. They do, however, provide a constraint on the physical structure of TMC1A disk due to their high upper energy level $\left(E_{\text {up }} \sim\right.$ $1000 \mathrm{~K})$. The peak flux density of each line has a $0.9 \mathrm{mJy}$ upper limit. For comparison, we also present the fundamental vibrational CO lines taken with CRIRES on the VLT (Herczeg et al. 2011 ) in the same figure with upper energy levels $\sim 3000 \mathrm{~K}$. These spectra are taken with a $0.2^{\prime \prime}$ slit at a PA of $64^{\circ}$, which is along the major axis of the Keplerian disk. A deep absorption is apparent at the systemic velocity of the coadded ${ }^{12} \mathrm{CO} v=1-0$ and ${ }^{13} \mathrm{CO} v=1-0$ spectra, and is similar to what we see in the rotational spectra in the ground vibrational state (Fig. 3). The broad blue absorption in the $\mathrm{CO}$ fundamental vibrational line is, meanwhile, a strong indication of a disk wind (Calvet et al. 1992; Pontoppidan et al. 2011; Bast et al. 2011; Herczeg et al. 2011).

In order to further constrain the $\mathrm{HCN} v_{2}=1$ emission, the weighted average of the two spectra $(e, f)$ is also shown in black in Fig. B.1. The stacked spectrum shows tantalizing features of beam diluted vibrational $\mathrm{HCN}$ emission. We expect that the emission should follow the ${ }^{13} \mathrm{CO} v=1-0$ line profile as the redshifted emission $\left(>8 \mathrm{~km} \mathrm{~s}^{-1}\right.$ ) comes from the hot surface layer of the disk instead of the disk wind (Herczeg et al. 2011). Therefore, we believe that the absorption feature in the stacked HCN spectrum and emission near the systemic velocity could be firmly detected with deeper observations.
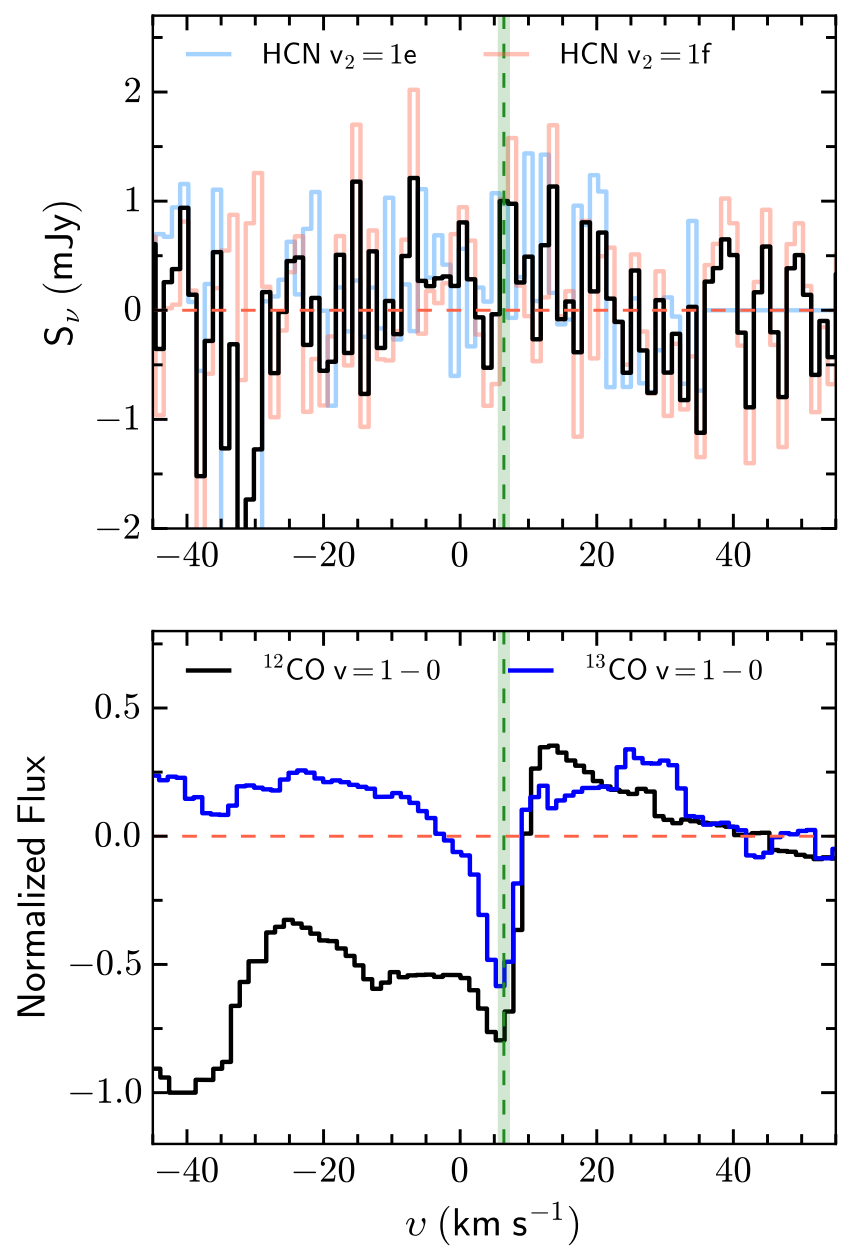

Fig. B.1. Top: HCN rovibrational spectra $\left(v_{2}=1 J=3-2 e / f\right)$ observed with ALMA. The spectra are extracted from the inner $1^{\prime \prime}$ region with red and blue denoting the $e$ and $f$ vibronic angular momentum quantum number, respectively. The black spectrum shows the stacked spectrum of the two lines, allowing a more stringent upper limit on the transition. Bottom: fundamental $v=1-0 \mathrm{CO}$ vibrational spectra taken with the VLT/CRIRES (Herczeg et al. 2011). The shown ${ }^{12} \mathrm{CO}$ spectrum is the co-added rotational line profiles from $R(3)$ to $P(9)$, while the ${ }^{13} \mathrm{CO}$ spectrum includes co-added $\mathrm{R}(10)$ to $\mathrm{P}(9)$. The green vertical solid line is the systemic velocity of the system, while the red horizontal dashed line indicates the baseline. 